\title{
Tropical Refuges with Exceptionally High Phylogenetic Diversity Reveal Contrasting Phylogenetic Structures
}

\author{
Lara Pugliesi and Alessandro Rapini \\ Departamento de Ciências Biológicas, Universidade Estadual de Feira de Santana, Avenida Transnordestina s/n, \\ Novo Horizonte, 44036-900 Feira de Santana, BA, Brazil \\ Correspondence should be addressed to Lara Pugliesi; larapugli@gmail.com
}

Received 15 September 2014; Revised 28 December 2014; Accepted 29 December 2014

Academic Editor: Stephan Koblmüller

Copyright (C) 2015 L. Pugliesi and A. Rapini. This is an open access article distributed under the Creative Commons Attribution License, which permits unrestricted use, distribution, and reproduction in any medium, provided the original work is properly cited.

\begin{abstract}
Loss of phylogenetic diversity (PD) has gained increasing attention in conservation biology. However, PD is not equally distributed in a phylogeny and can be better assessed when species relatedness (phylogenetic structure: PS) is also considered. Here, we investigate PD and PS in two refuges of biodiversity in northeastern Brazil: the Bahia Costal Forest (BCF) in the Atlantic Forest domain and Chapada Diamantina (CD) in the Caatinga domain. We used geographic data of 205 species at two spatial scales and a chronogram of Apocynaceae based on $m a t K$ sequences to estimate PD and PS. Our results show an exceptionally high PD in both refuges, overdispersed in BCF and clustered in $\mathrm{CD}$, although this difference is less evident or absent for recent relationships, especially at a smaller spatial scale. Overall, PS suggests long-term competitive exclusion under climatic stability, currently balanced by habitat filtering, in BCF, and biome conservatism and limited dispersal leading to in situ diversification and high density of microendemics in CD. The phylogenetically clustered flora in $\mathrm{CD}$, also threatened by climate changes, are naturally more vulnerable than BCF. Therefore, while in situ conservation may ensure protection of biodiversity in BCF, emergency ex situ conservation is strongly recommended in $\mathrm{CD}$.
\end{abstract}

\section{Introduction}

Currently, the consensus is that biodiversity loss reduces community efficiency, stability, and productiveness [1], and the best strategy for biological conservation is through gains in phylogenetic diversity (PD) [2,3]. Species do not contribute equally to total PD of an area but with their distinct evolutionary history $[4,5]$. Closely related species, sharing a great extent of evolutionary history, are more likely to be redundant, whereas distantly related species are expected to play different ecological functions and provide different goods and services. Therefore, PD, based on the sum of branch length, is an important measure in conservation biology [5-7]. Species loss diminishes PD, but PD loss cannot be directly predicted by species loss, because proportions of PD loss may be higher than proportions of species loss when extinctions are clumped or biased to relictual lineages. Accordingly, communities whose species composition is phylogenetically clustered tend to lose evolutionary diversity more quickly, whereas communities in which species are phylogenetically overdispersed tend to lose less evolutionary diversity during extinctions $[8,9]$. Therefore, PD loss depends on how communities are phylogenetically structured (species relatedness).

Phylogenetic structure (PS) represents the overall relatedness in a species assembly (e.g., [10]) and combines community ecology and evolutionary thinking [11] into an interdisciplinary approach, community phylogenetics or ecophylogenetics [12]. PS is obtained by comparing the community phylogenetic distance to a null model, which randomize variants, such as species relationships and distributions $[10,11]$. This metric differs from PD, which represents only the sum of phylogenetic distances of a community [5] and does not provide information on how species of this community are phylogenetically related. According to PS, communities may be phylogenetically clustered, which indicates cooccurrence of closely related species and suggests a stronger influence of an environmental filter on the community. In contrast, 
phylogenetically overdispersed communities indicate local exclusion of closely related species and suggest a stronger influence of interspecific competition and/or other densitydependent negative interactions [11]. These interpretations are mainly supported by a strong tendency towards phylogenetic niche conservatism (PNC) during diversifications, as shown by Crisp et al. [13] for plants. According to PNC, closely related species tend to share traits and occupy similar habitats [14]. However, PS is scale and context dependent, and alternative interpretations can emerge from similar patterns, often being equivocal when traits are not taken into account. For instance, clustered phylogenies can also result from character displacements among closely related species allowing their coexistence or from limited dispersal and in situ speciation. On the other hand, overdispersed phylogenies may also result from convergent ecological traits among distantly related species. Finally, unstructured phylogenies suggest a balance between environmental constraints and biological interactions or a prevalent influence of neutral processes, such as a stochastic dynamic of dispersal, speciation, and extinction, rather than niche-based processes (e.g., [3, 11, 1519]). Furthermore, null models used for assessing PS can also affect results and eventually confer spurious structures for unstructured phylogenies (e.g., [16, 20-22]).

Ecophylogenetics still lacks a consistent conceptual framework [12] and the use of PD as a proxy for functional diversity has been criticized for lacking empirical evidence [23]. However, PD and PS are complementary measures of biodiversity and can be properly used for biogeography, ecology, and conservation biology. Phylogenetics has been used to assess historical and ecological drivers at different spatial scales $[12,18]$, from latitudinal gradient of species richness (SR) [24], biogeographic processes during the Cenozoic [25], and coastal dune ecosystem [26] at a global scale to habitat heterogeneity [27, 28], successional pathways [29], and altitudinal gradient [30] at regional or, mainly, local scales. Although Brazil harbours the richest flora in the world, with more than 32,000 species of angiosperms [31], studies applying phylogeny to interpret plant composition (e.g., [32, 33]) are still scarce.

Here, we investigate PD and PS in two centres of biodiversity (rich in species and endemisms), the Chapada Diamantina and the Bahia Costal Forest. Although only $\sim 120 \mathrm{~km}$ separates one from the other, these centres are under different environmental conditions and floristic domains and, together, comprise most of the angiosperm diversity in northeastern Brazil (Figure 1).

Chapada Diamantina (CD) is the largest continuous plateau in the northern Espinhaço Range. Above $900 \mathrm{~m}$, particularly in the south and east, the plateau is covered by rocky fields (Campos Rupestres), an open vegetation biome associated with quartzite outcrops, rich in plant species and endemisms (e.g., [34]). It is floristically influenced by seasonally dry forests from the surrounding Caatinga but is also home of palaeomicroendemics [35] and was recently postulated as a historical refuge for fire-sensitive lineages [36]. The Bahia Costal Forest (BCF) ecoregion [37] is the largest area of climatic stability in the northern block of the Atlantic Forest [38-40]. Ranging from northern Espirito

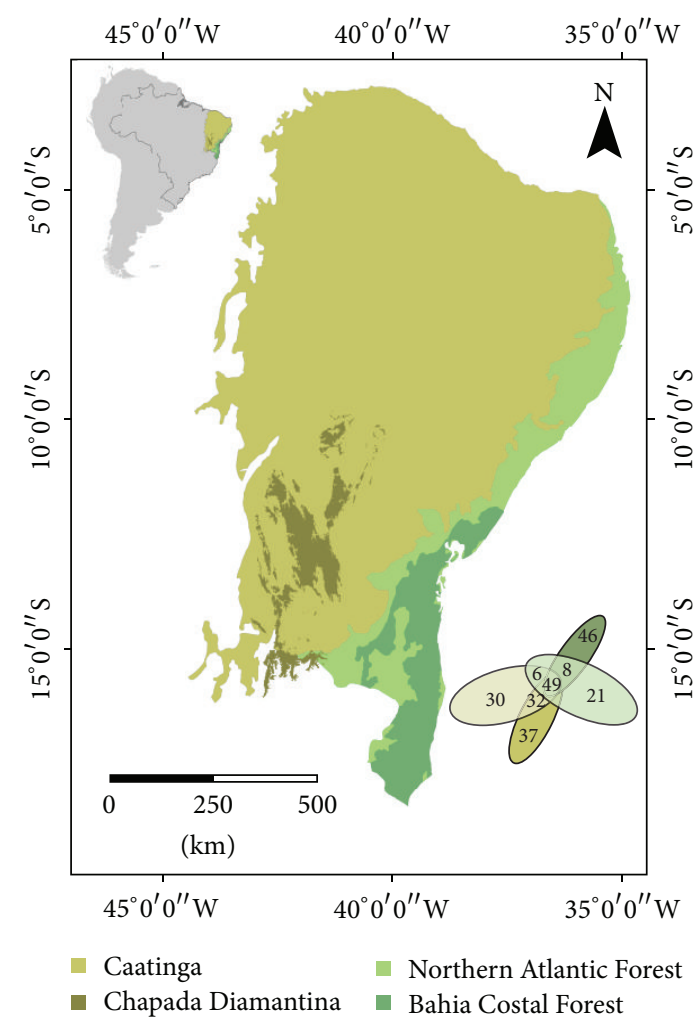

FIgURE 1: Northeastern Brazil in South America, showing the number of Apocynaceae species per region in a Venn diagram.

Santo to southern Bahia, it is dominated by evergreen forests, harbouring a forest refuge, with high levels of endemism [41] and one of the highest tree species densities in the world [42]. Since CD and BCF are species rich, high PD values are expected for both. However, more species of shrubs and lianas are found in CD whereas BCF is richer in species of trees. This difference probably affected species relatedness and community resilience. Therefore, assessing PS in CD and BCF may provide important basis for phyloconservation policies to protect the high biodiversity found in these areas.

For assessing PS in CD and BCF, we used Apocynaceae and ecophylogenetics in a macroecological approach. The Apocynaceae are one of the ten largest families of angiosperms and their SR distribution shows the highest correlation with angiosperm SR distribution in Brazil, when the five richest angiosperm families in the country are taken into account (Figure 2). This high correlation strongly confirms the Apocynaceae to be a good indicator for plant diversity across the Brazilian territory. The Apocynaceae consist of approximately 5,000 species [43] and 360 genera [44] and are also well represented in Brazil, with 770 species and 73 genera [45]. They are latescent plants with pentamerous, gamopetalous, isostemonous, bicarpelar flowers and comprise a broad range of habits (trees, shrubs, herbs, and lianas) and have pollen transferred as monads, tetrads, or in pollinia, berry-like or bifollicular fruits and seeds with or without coma. The family is widespread over the world, especially in tropical and subtropical regions, and occurs 


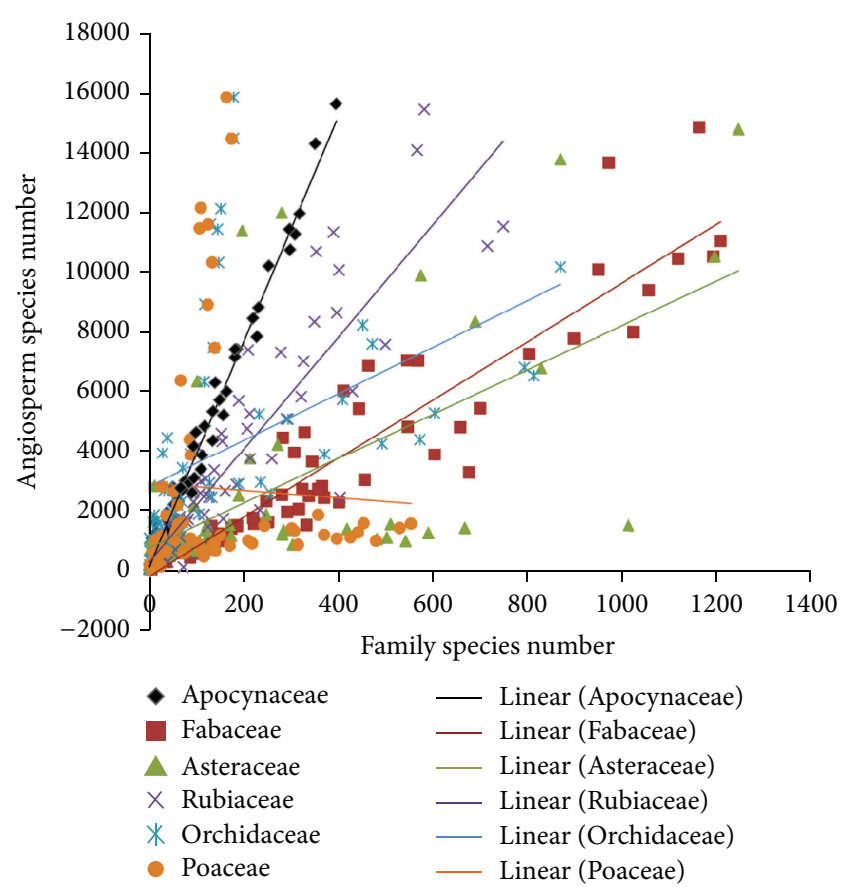

FIgURE 2: Family species number $\times$ angiosperm species number per vegetation, states, and regions of Brazil. Spearman's correlation values for Apocynaceae and the five richest families of angiosperm in Brazil [31]: Apocynaceae, $r_{s}=0.98(P<2.2 e-16)$; Fabaceae, $r_{s}=0.95(P<2.2 e-16)$; Rubiaceae, $r_{s}=0.88(P<2.2 e-16)$; Orchidaceae, $r_{s}=0.81(P=5.47 e-15)$; Asteraceae, $r_{s}=0.71$ $(P=3.29 e-10)$; and Poaceae, $r_{s}=0.28(P=0.03)$.

in almost any habitat, from lowland wet forests to deserts and grasslands in high altitudes [46, 47]. In Brazil, such diversity is classified in three subfamilies: Apocynoideae, Asclepiadoideae, and Rauvolfioideae [47]. Asclepiadoideae comprises a clade that consists mainly of shrubs and lianas, and $\mathrm{CD}$ is an important centre of diversity of the subfamily. Together, Apocynoideae and Rauvolfioideae comprise the early diverging lineages of Apocynaceae; the former consists mainly of shrubs and lianas, whereas trees are prevalent in the latter subfamily. Apocynoideae and Rauvolfioideae form a basal grade in Apocynaceae and their species are usually broadly distributed or inhabit predominantly tropical forests such as BCF.

In this study, we map the Apocynaceae PD in northeastern Brazil to evaluate whether $\mathrm{CD}$ and $\mathrm{BCF}$ present exceptionally high PD and compare the Apocynaceae PS in the two areas. Taking into account the ecological specificities across the Apocynaceae phylogeny, we would expect to find clustered communities in both $\mathrm{CD}$ and BCF but concentrated in different lineages. The most derived Asclepiadoideae would be concentrated in $\mathrm{CD}$ whereas lineages of the Rauvolfioideae-Apocynoideae basal grade would be mainly concentrated in BCF. We then try to identify ecological and evolutionary factors that may have affected plant community in the two areas and suggest general perspectives for conserving the biodiversity in both.

\section{Material and Methods}

We built a database with approximately 7,000 specimens, representing 205 species and 47 genera of Apocynaceae native to the Caatinga and Atlantic Forest domains in northeast Brazil (Table 1) based on exsiccates from the main herbaria in Brazil, Europe, and the United States. GPS coordinates were extracted from labels and confirmed or recovered with the help of Google Earth. Specimens without locality were treated at the municipal headquarter.

A calibrated phylogeny was constructed with matK sequences of 142 species of Apocynaceae and five from the Loganiaceae (outgroup) from Genbank (the appendix). We sampled 95 genera, representing the five subfamilies and most tribes of Apocynaceae. Sequences were initially aligned in Muscle [48] and subsequently manually adjusted in mesquite [49]. Age estimates were obtained using BEAST 1.8 [50] as implemented in CIPRES [51], using a GTR substitution model, gamma distribution, and relaxed molecular clock. The analysis was conducted from a random starting tree, with a Yule speciation model. Dating was calibrated using two fossils: a comose seed (Apocynospermum) from the Eocene $($ mean $=1.5$, Std.Dev. $=1$, and Offset $=47$, Lognormal prior) assigned to the APSA clade stem node (Apocynoideae, Periplocoideae, Secamonoideae, and Asclepiadoideae) [52] and a tetrad (Polyporotetradites laevigatus) from the Oligocene/Miocene boundary (mean $=1.5$, Std.Dev. $=1$, and Offset $=23$, Lognormal prior) assigned to Tacazzea (Periplocoideae stem node) [53]. A Monte Carlo-Markov chain was run for $5 \times 10^{7}$ generations, saving a tree every 2,000 generations. The log file was analysed in TRACER 1.6 [54] to assess whether the effective sample size reached 200 for all parameters. The maximum credibility tree was recovered in TreeAnnotator 1.8.0 [54], after deleting the first $10 \%$ of saved trees (burn-in).

We assessed Apocynaceae PD and PS using a pseudochronogram constructed from the calibrated tree, in which species branch length was treated as an average of total lineage branch length. We estimated the total branch length of a lineage assuming half-aged, successive, and balanced dichotomies. Accordingly, species were added regularly at the middistance of the longest branch of that lineage and the sum of lineage branch lengths was divided by the number of species of the lineage in northeastern Brazil (Figure 3). Eight genera lacking molecular data were included based on their taxonomic position and/or other molecular markers [47].

Since biodiversity metrics may be strongly affected by scales, we calculated SR, PD, and PS for $0.5^{\circ} \times 0.5^{\circ}$ and $0.08^{\circ} \times$ $0.08^{\circ}$ grids to test the consistence of observed patterns under different spatial scales. PD, after standardization of species branch length, and SR were calculated using Biodiverse v. 0.19 [55]. We estimated PS through net relatedness index (NRI) and nearest taxon index (NTI) for CD and BCF based on cells with exceptionally high PD (higher than $95 \%$ of cells for $05^{\circ} \times 05^{\circ}$ grid and higher than $97.5 \%$ for $0.08^{\circ} \times 0.08^{\circ}$ grid) using Phylocom 4.2 [56]. Null communities were generated adopting a model that randomizes species relationships keeping their original species richness $[22,57]$. Species abundance was not considered because our data is based on herbarium 
TABLE 1: Native species of Apocynaceae from northeastern Brazil (Caatinga and Atlantic Forest domains in the Northeast Brazil), indicating species occurring in the Caatinga and Atlantic forest. CD denotes species occurring in Chapada Diamantina and BCF in Bahia Costal Forest, in bold when they are endemic to these regions and with an asterisk when they appear in $0.08^{\circ} \times 0.08^{\circ}$ cells with exceptionally high phylogenetic diversity, considering one neighborhood cell.

\begin{tabular}{|c|c|c|}
\hline \multirow{2}{*}{ Species } & \multicolumn{2}{|c|}{ Floristic domains } \\
\hline & Caatinga & Atlantic Forest \\
\hline Allamanda blanchetii A.DC. & $\mathrm{CD}^{*}$ & \\
\hline Allamanda calcicola Souza-Silva \& Rapini & $\mathrm{x}$ & \\
\hline Allamanda cathartica L. & & BCF $^{*}$ \\
\hline Allamanda doniana Müll.Arg. & $\mathrm{x}$ & $\mathrm{BCF}^{*}$ \\
\hline Allamanda martii Müll.Arg. & & $\mathrm{BCF}^{*}$ \\
\hline Allamanda puberula A.DC. & $\mathrm{CD}^{*}$ & $\mathrm{BCF}$ \\
\hline Allamanda thevetifolia Müll.Arg. & $\mathrm{x}$ & \\
\hline Araujia sericifera Brot. & & $\mathrm{x}$ \\
\hline Asclepias candida Vell. & $\mathrm{CD}^{*}$ & \\
\hline Asclepias curassavica $\mathrm{L}$. & $\mathrm{CD}^{*}$ & BCF $^{*}$ \\
\hline Asclepias mellodora A.St.-Hil. & $\mathrm{CD}^{*}$ & $\mathrm{BCF}^{*}$ \\
\hline Aspidosperma cuspa S.F.Blake ex Pittier & $\mathrm{CD}$ & \\
\hline Aspidosperma cylindrocarpon Müll.Arg. & & $\mathrm{x}$ \\
\hline Aspidosperma discolor A.DC. & $\mathrm{CD}^{*}$ & $\mathrm{BCF}^{*}$ \\
\hline Aspidosperma illustre (Vell.) Kuhlm. \& Pirajá & $\mathrm{x}$ & $\mathrm{BCF}$ \\
\hline Aspidosperma limae Woodson & & $\mathrm{x}$ \\
\hline Aspidosperma macrocarpon Mart. & $\mathrm{CD}$ & \\
\hline Aspidosperma multiflorum A.DC. & $\mathrm{x}$ & $\mathrm{x}$ \\
\hline Aspidosperma parvifolium A.DC. & $\mathrm{CD}^{*}$ & $\mathrm{BCF}^{*}$ \\
\hline Aspidosperma polyneuron Müll.Arg. & $\mathrm{CD}^{*}$ & $\mathrm{BCF}$ \\
\hline Aspidosperma pyricollum Müll.Arg. & & $\mathrm{x}$ \\
\hline Aspidosperma pyrifolium Mart. & $\mathrm{CD}^{*}$ & $\mathrm{BCF}^{*}$ \\
\hline Aspidosperma ramiflorum Müll.Arg. & & $\mathrm{BCF}$ \\
\hline Aspidosperma schultesii Woodson & & $\mathrm{BCF}$ \\
\hline Aspidosperma spruceanum Benth. ex Müll.Arg. & $\mathrm{CD}^{*}$ & $\mathrm{BCF}^{*}$ \\
\hline Aspidosperma subincanum Mart. & $\mathrm{x}$ & \\
\hline Aspidosperma thomasii Marc.-Ferr. & & BCF $^{*}$ \\
\hline Aspidosperma tomentosum Mart. & $\mathrm{CD}^{*}$ & $\mathrm{x}$ \\
\hline Aspidosperma ulei Markgr. & $\mathrm{x}$ & \\
\hline Bahiella blanchetii (A.DC.) J.F.Morales & & BCF $^{*}$ \\
\hline Bahiella infundibuliflora J.F.Morales & & BCF \\
\hline Barjonia chlorifolia Decne. & $\mathrm{CD}^{*}$ & \\
\hline Barjonia erecta (Vell.) K.Schum. & $\mathrm{CD}^{*}$ & \\
\hline Barjonia glazioui Marquete & $\mathrm{CD}^{*}$ & \\
\hline Blepharodon ampliflorum E.Fourn. & $\mathrm{CD}^{*}$ & $\mathrm{x}$ \\
\hline Blepharodon bicolor Decne. & $\mathrm{CD}$ & \\
\hline Blepharodon costae Fontella \& Morillo & & $\mathrm{BCF}^{*}$ \\
\hline Blepharodon manicatum (Decne.) Fontella & $\mathrm{CD}^{*}$ & \\
\hline Blepharodon pictum (Vahl) W.D.Stevens & $\mathrm{CD}^{*}$ & $\mathrm{BCF}^{*}$ \\
\hline Condylocarpon intermedium Müll.Arg. & & $\mathrm{BCF}^{*}$ \\
\hline Condylocarpon isthmicum (Vell.) A.DC. & $\mathrm{CD}^{*}$ & BCF $^{*}$ \\
\hline Couma rigida Müll.Arg. & $\mathrm{CD}^{*}$ & $\mathrm{BCF}^{*}$ \\
\hline Cynanchum montevidense Spreng. & $\mathrm{x}$ & $\mathrm{x}$ \\
\hline Cynanchum roulinioides (E.Fourn.) Rapini & $\mathrm{CD}^{*}$ & \\
\hline
\end{tabular}


TABle 1: Continued.

\begin{tabular}{|c|c|c|}
\hline \multirow{2}{*}{ Species } & \multicolumn{2}{|c|}{ Floristic domains } \\
\hline & Caatinga & Atlantic Forest \\
\hline Ditassa arianeae Fontella \& E.A.Schwarz & $\mathrm{x}$ & $\mathrm{BCF}^{*}$ \\
\hline Ditassa blanchetii Decne. & & $\mathrm{BCF}^{*}$ \\
\hline Ditassa capillaris E.Fourn. & $\mathrm{CD}^{*}$ & $\mathrm{BCF}^{*}$ \\
\hline Ditassa congesta E.Fourn. & $\mathrm{x}$ & \\
\hline Ditassa crassifolia Decne. & $\mathrm{CD}$ & $\mathrm{BCF}^{*}$ \\
\hline Ditassa dardanoi T.U.P.Konno \& Wand. & $\mathrm{CD}$ & \\
\hline Ditassa dolichoglossa Schltr. & $\mathrm{x}$ & \\
\hline Ditassa glazioui E.Fourn. & $\mathrm{CD}^{*}$ & $\mathrm{x}$ \\
\hline Ditassa grandiflora E.Fourn. & $\mathrm{CD}^{*}$ & \\
\hline Ditassa hastata Decne. & $\mathrm{CD}^{*}$ & $\mathrm{x}$ \\
\hline Ditassa hispida (Vell.) Fontella & $\mathrm{CD}$ & $\mathrm{BCF}^{*}$ \\
\hline Ditassa lenheirensis Silveira & $\mathrm{CD}^{*}$ & $\mathrm{x}$ \\
\hline Ditassa melantha Silveira & $\mathrm{CD}^{*}$ & \\
\hline Ditassa obcordata Mart. & $\mathrm{CD}^{*}$ & \\
\hline Ditassa oxyphylla Turcz. & $\mathrm{CD}^{*}$ & $\mathrm{BCF}$ \\
\hline Ditassa pohliana E.Fourn. & $\mathrm{CD}^{*}$ & $\mathrm{BCF}$ \\
\hline Ditassa retusa Mart. & $\mathrm{CD}^{*}$ & $\mathrm{x}$ \\
\hline Ditassa rotundifolia (Decne.) Baill. ex K.Schum. & $\mathrm{CD}^{*}$ & $\mathrm{x}$ \\
\hline Ditassa succedanea Rapini & $\mathrm{CD}^{*}$ & \\
\hline Fischeria stellata (Vell.) E.Fourn. & $\mathrm{CD}$ & $\mathrm{BCF}$ \\
\hline Forsteronia australis Müll.Arg. & $\mathrm{CD}^{*}$ & BCF \\
\hline Forsteronia glabrescens Müll.Arg. & $\mathrm{x}$ & \\
\hline Forsteronia leptocarpa (Hook. \& Arn.) A.DC. & $\mathrm{CD}$ & $\mathrm{BCF}^{*}$ \\
\hline Forsteronia montana Müll.Arg. & & $\mathrm{BCF}^{*}$ \\
\hline Forsteronia pubescens A.DC. & $\mathrm{CD}^{*}$ & $\mathrm{x}$ \\
\hline Forsteronia rufa Müll.Arg. & $\mathrm{CD}^{*}$ & $\mathrm{BCF}$ \\
\hline Forsteronia thyrsoidea Müll.Arg. & $\mathrm{CD}^{*}$ & $\mathrm{BCF}$ \\
\hline Funastrum clausum (Jacq.) Schltr. & $\mathrm{CD}^{*}$ & $\mathrm{BCF}^{*}$ \\
\hline Geissospermum laeve (Vell.) Miers & & $\mathrm{BCF}^{*}$ \\
\hline Gonolobus parviflorus Decne. & $\mathrm{x}$ & $\mathrm{BCF}^{*}$ \\
\hline Gonolobus rostratus (Vahl) R.Br. ex Shult. & $\mathrm{x}$ & \\
\hline Hancornia speciosa Gomes & $\mathrm{CD}^{*}$ & BCF $^{*}$ \\
\hline Hemipogon carassensis (Malme) Rapini & $\mathrm{CD}^{*}$ & \\
\hline Himatanthus bracteatus (A. DC.) Woodson & $\mathrm{CD}^{*}$ & $\mathrm{BCF}^{*}$ \\
\hline Himatanthus drasticus (Mart.) Plumel & $\mathrm{CD}^{*}$ & $\mathrm{BCF}$ \\
\hline Himatanthus obovatus (Müll. Arg.) Woodson & $\mathrm{CD}$ & $\mathrm{BCF}^{*}$ \\
\hline Himatanthus phagedaenicus (Mart.) Woodson & & $\mathrm{x}$ \\
\hline Jobinia connivens (Hook. \& Arn.) Malme & $\mathrm{CD}^{*}$ & \\
\hline Jobinia lindbergii E.Fourn. & $\mathrm{CD}^{*}$ & \\
\hline Lacmellea bahiensis J.F.Morales & & BCF $^{*}$ \\
\hline Lacmellea pauciflora (Kuhlm.) Markgr. & & BCF \\
\hline Macoubea guianensis Aubl. & & $\mathrm{BCF}^{*}$ \\
\hline Macroditassa laurifolia (Decne.) Fontella & $\mathrm{CD}^{*}$ & $\mathrm{BCF}^{*}$ \\
\hline Malouetia cestroides (Nees ex Mart.) Müll.Arg. & & BCF $^{*}$ \\
\hline Mandevilla alexicaca (Mart. ex Stadelm.) M.F.Sales & $\mathrm{CD}^{*}$ & \\
\hline
\end{tabular}


TABle 1: Continued.

\begin{tabular}{|c|c|c|}
\hline \multirow{2}{*}{ Species } & \multicolumn{2}{|c|}{ Floristic domains } \\
\hline & Caatinga & Atlantic Forest \\
\hline Mandevilla bahiensis (Woodson) M.F.Sales \& Kin.-Gouv. & $\mathrm{CD}^{*}$ & BCF $^{*}$ \\
\hline Mandevilla catimbauensis-Silva et al. & $\mathrm{x}$ & \\
\hline Mandevilla dardanoi M.F.Sales et al. & $\mathrm{x}$ & $\mathrm{x}$ \\
\hline Mandevilla emarginata (Vell.) C.Ezcurra & $\mathrm{CD}^{*}$ & \\
\hline Mandevilla fistulosa M.F.Sales et al. & & $\mathrm{BCF}$ \\
\hline $\begin{array}{l}\text { Mandevilla guanabarica Casar. ex M.F.Sales, Kin.-Gouv. \& } \\
\text { A.O.Simões }\end{array}$ & & $\mathrm{BCF}$ \\
\hline Mandevilla hatschbachii M.F.Sales et al. & CD & \\
\hline Mandevilla hirsuta (A.Rich.) K.Schum. & $\mathrm{CD}^{*}$ & $\mathrm{BCF}$ \\
\hline Mandevilla illustris (Vell.) Woodson & $\mathrm{CD}^{*}$ & $\mathrm{x}$ \\
\hline Mandevilla leptophylla (A.DC.) K.Schum. & $\mathrm{CD}^{*}$ & \\
\hline Mandevilla longiflora (Desf.) Pichon & $\mathrm{CD}^{*}$ & \\
\hline Mandevilla luetzelburgii Woodson & $\mathrm{CD}$ & $\mathrm{BCF}$ \\
\hline Mandevilla martiana (Stadelm.) Woodson & $\mathrm{CD}^{*}$ & \\
\hline Mandevilla martii (Müll.Arg.) Pichon & $\mathrm{CD}$ & \\
\hline Mandevilla microphylla (Stadelm.) M.F.Sales \& Kin.-Gouv. & $\mathrm{CD}^{*}$ & BCF $^{*}$ \\
\hline Mandevilla moricandiana (A.DC.) Woodson & $\mathrm{CD}^{*}$ & $\mathrm{BCF}^{*}$ \\
\hline Mandevilla myriophylla (Taub. ex Ule) Woodson & $\mathrm{CD}$ & \\
\hline Mandevilla permixta Woodson & & $\mathbf{B C F}^{*}$ \\
\hline Mandevilla sancta (Stadelm.) Woodson & $\mathrm{CD}^{*}$ & $\mathrm{x}$ \\
\hline $\begin{array}{l}\text { Mandevilla scabra (Hoffmanns. ex Roem. \& Schult.) } \\
\text { K.Schum. }\end{array}$ & $\mathrm{CD}^{*}$ & BCF $^{*}$ \\
\hline Mandevilla tenuifolia (J.C.Mikan) Woodson & $\mathrm{CD}^{*}$ & $\mathrm{x}$ \\
\hline Marsdenia altissima (Jacq.) Dugand & $\mathrm{CD}^{*}$ & $\mathrm{BCF}$ \\
\hline Marsdenia caatingae Morillo & $\mathrm{x}$ & $\mathrm{BCF}^{*}$ \\
\hline Marsdenia carvalhoi Morillo \& Carnevali & & BCF \\
\hline Marsdenia dorothyae Fontella \& Morillo & & $\mathrm{BCF}^{*}$ \\
\hline Marsdenia heringeri Fontella & $\mathrm{x}$ & \\
\hline Marsdenia hilariana E.Fourn. & $\mathrm{CD}^{*}$ & $\mathrm{BCF}$ \\
\hline Marsdenia loniceroides E.Fourn. & $\mathrm{x}$ & $\mathrm{x}$ \\
\hline $\begin{array}{l}\text { Marsdenia macrophylla (Humb. \& Bonpl. ex Schult.) } \\
\text { E.Fourn. }\end{array}$ & $\mathrm{x}$ & \\
\hline Marsdenia megalantha Goyder \& Morillo & $\mathrm{x}$ & \\
\hline Marsdenia pickelii Fontella \& Morillo & $\mathrm{x}$ & \\
\hline Marsdenia queirozii Fontella & $\mathrm{x}$ & \\
\hline Marsdenia suberosa (E.Fourn.) Malme & $\mathrm{CD}^{*}$ & $\mathrm{BCF}$ \\
\hline Marsdenia zehntneri Fontella & $\mathrm{CD}$ & \\
\hline Matelea bahiensis Morillo \& Fontella & & BCF $^{*}$ \\
\hline Matelea denticulata (Vahl) Fontella \& E.A.Schwarz & $\mathrm{x}$ & $\mathrm{BCF}^{*}$ \\
\hline Matelea endressiae Fontella \& Goes & $\mathrm{CD}$ & \\
\hline Matelea ganglinosa (Vell.) Rapini & $\mathrm{CD}^{*}$ & $\mathrm{BCF}^{*}$ \\
\hline Matelea harleyi Fontella \& Morillo & $\mathrm{CD}$ & \\
\hline Matelea morilloana Fontella & $\mathrm{CD}^{*}$ & \\
\hline Matelea nigra (Decne.) Morillo \& Fontella & $\mathrm{CD}^{*}$ & \\
\hline Matelea orthosioides (E.Fourn.) Fontella & $\mathrm{CD}^{*}$ & $\mathrm{BCF}^{*}$ \\
\hline Matelea pedalis (E.Fourn.) Fontella \& E.A.Schwarz & $\mathrm{CD}^{*}$ & \\
\hline
\end{tabular}


TABle 1: Continued.

\begin{tabular}{|c|c|c|}
\hline \multirow{2}{*}{ Species } & \multicolumn{2}{|c|}{ Floristic domains } \\
\hline & Caatinga & Atlantic Forest \\
\hline Matelea riparia Morillo & & BCF \\
\hline Matelea roulinioides Agra \& W.D.Stevens & $\mathrm{x}$ & \\
\hline Matelea santosii Morillo \& Fontella & & BCF \\
\hline Metastelma giuliettianum Fontella & $\mathrm{CD}^{*}$ & \\
\hline Metastelma harleyi Fontella & $\mathrm{CD}^{*}$ & \\
\hline Metastelma myrtifolium Decne. & $\mathrm{CD}^{*}$ & \\
\hline Minaria acerosa (Mart.) T.U.P.Konno \& Rapini & $\mathrm{CD}^{*}$ & \\
\hline Minaria cordata (Turcz.) T.U.P.Konno \& Rapini & $\mathrm{CD}^{*}$ & $\mathrm{x}$ \\
\hline Minaria decussata (Mart.) T.U.P.Konno \& Rapini & $\mathrm{CD}$ & \\
\hline $\begin{array}{l}\text { Minaria harleyi (Fontella \& Marquete) Rapini \& } \\
\text { U.C.S.Silva }\end{array}$ & $\mathrm{CD}^{*}$ & \\
\hline Minaria volubilis Rapini \& U.C.S.Silva & $\mathrm{CD}^{*}$ & \\
\hline Monsanima morrenioides (Goyder) Liede \& Meve & $\mathrm{CD}^{*}$ & \\
\hline Nephradenia asparagoides (Decne.) E.Fourn. & $\mathrm{CD}^{*}$ & \\
\hline Odontadenia hypoglauca Müll.Arg. & $\mathrm{x}$ & \\
\hline Odontadenia lutea (Vell.) Markgr. & $\mathrm{CD}^{*}$ & BCF $^{*}$ \\
\hline Orthosia parviflora (E.Fourn.) Liede \& Meve & & $\mathrm{BCF}^{*}$ \\
\hline Orthosia scoparia (Nutt.) Liede \& Meve & $\mathrm{x}$ & \\
\hline Oxypetalum arachnoideum E.Fourn. & $\mathrm{CD}^{*}$ & \\
\hline Oxypetalum banksii R.Br. ex Schult. & $\mathrm{CD}$ & $\mathrm{BCF}^{*}$ \\
\hline Oxypetalum capitatum Mart. & $\mathrm{CD}^{*}$ & \\
\hline Oxypetalum erostre E.Fourn. & $\mathrm{CD}^{*}$ & \\
\hline Oxypetalum harleyi (Fontella \& Goyder) Farinaccio & $\mathrm{CD}^{*}$ & $\mathrm{BCF}$ \\
\hline Oxypetalum jacobinae Decne. & $\mathrm{CD}^{*}$ & BCF \\
\hline Oxypetalum laciniatum Rapini \& Farinaccio & & BCF \\
\hline Oxypetalum montanum Mart. & $\mathrm{CD}^{*}$ & \\
\hline Oxypetalum pachyglossum Decne. & & $\mathrm{BCF}^{*}$ \\
\hline Oxypetalum strictum Mart. & $\mathrm{CD}^{*}$ & \\
\hline Oxypetalum warmingii (E.Fourn.) Fontella \& Marquete & $\mathrm{x}$ & \\
\hline Peltastes peltatus (Vell.) Woodson & & $\mathrm{BCF}^{*}$ \\
\hline Peltastes pulcher (Miers) J.F.Morales & & $\mathrm{BCF}^{*}$ \\
\hline Peplonia adnata (E.Fourn.) U.C.S.Silva \& Rapini & $\mathrm{CD}^{*}$ & $\mathrm{BCF}$ \\
\hline Peplonia asteria (Vell.) Fontella \& E.A.Schwarz & & $\mathrm{BCF}^{*}$ \\
\hline Peplonia axillaris (Vell.) Fontella \& Rapini & & BCF \\
\hline $\begin{array}{l}\text { Peplonia bradeana (Fontella \& E.A.Schwarz) Fontella \& } \\
\text { Rapini }\end{array}$ & & $\mathrm{BCF}^{*}$ \\
\hline Peplonia macrophylla (Malme) U.C.S.Silva \& Rapini & $\mathrm{CD}^{*}$ & \\
\hline Petalostelma cearense Malme & $\mathrm{x}$ & \\
\hline Petalostelma dardanoi Fontella & $\mathrm{x}$ & \\
\hline Petalostelma martianum (Decne.) E.Fourn. & $\mathrm{x}$ & \\
\hline Prestonia annularis G.Don & & BCF \\
\hline Prestonia bahiensis Müll.Arg. & $\mathrm{CD}^{*}$ & $\mathrm{BCF}^{*}$ \\
\hline Prestonia calycina Müll.Arg. & $\mathrm{x}$ & BCF \\
\hline Prestonia coalita (Vell.) Woodson & $\mathrm{CD}^{*}$ & BCF* $^{*}$ \\
\hline Prestonia didyma (Vell.) Woodson & & BCF $^{*}$ \\
\hline
\end{tabular}


TABle 1: Continued.

\begin{tabular}{|c|c|c|}
\hline \multirow{2}{*}{ Species } & \multicolumn{2}{|c|}{ Floristic domains } \\
\hline & Caatinga & Atlantic Forest \\
\hline Prestonia erecta (Malme) J.F.Morales & $\mathrm{CD}$ & \\
\hline Prestonia lagoensis (Müll.Arg.) Woodson & $\mathrm{CD}^{*}$ & \\
\hline Prestonia quinquangularis (Jacq.) Spreng. & & BCF $^{*}$ \\
\hline Rauvolfia atlantica Emygdio & & BCF $^{*}$ \\
\hline Rauvolfia bahiensis A.DC. & & BCF $^{*}$ \\
\hline Rauvolfia grandiflora Mart. & $\mathrm{x}$ & BCF \\
\hline Rauvolfia ligustrina Willd. & $\mathrm{x}$ & $\mathrm{x}^{*}$ \\
\hline Rauvolfia mattfeldiana Markgr. & $\mathrm{CD}$ & \\
\hline Rauvolfia moricandii A.DC. & & $\mathrm{BCF}^{*}$ \\
\hline Rauvolfia paucifolia A.DC. & $\mathrm{CD}$ & \\
\hline Schubertia grandiflora Mart. & $\mathrm{CD}^{*}$ & \\
\hline Schubertia morilloana Fontella & $\mathrm{CD}^{*}$ & \\
\hline Schubertia multiflora Mart. & $\mathrm{CD}^{*}$ & $\mathrm{BCF}$ \\
\hline Secondatia densiflora A.DC. & $\mathrm{CD}^{*}$ & $\mathrm{x}$ \\
\hline Secondatia floribunda A.DC. & $\mathrm{CD}^{*}$ & $\mathrm{BCF}^{*}$ \\
\hline Skytanthus hancorniifolius (A.DC.) Miers & $\mathrm{CD}^{*}$ & $\mathrm{x}$ \\
\hline Stenomeria decalepis Turcz. & & $\mathrm{BCF}^{*}$ \\
\hline Stipecoma peltigera (Stadelm.) Müll.Arg. & $\mathrm{CD}^{*}$ & $\mathrm{x}$ \\
\hline Tabernaemontana catharinensis A.DC. & $\mathrm{x}$ & $\mathrm{x}$ \\
\hline Tabernaemontana flavicans Willd. ex Roem. \& Schult. & & $\mathrm{BCF}^{*}$ \\
\hline Tabernaemontana grandiflora $\mathrm{L}$. & & $\mathrm{BCF}$ \\
\hline Tabernaemontana hystrix Steud. & & $\mathrm{BCF}$ \\
\hline Tabernaemontana laeta Mart. & $\mathrm{x}$ & $\mathrm{BCF}$ \\
\hline Tabernaemontana salzmannii A.DC. & & $\mathrm{BCF}^{*}$ \\
\hline Tabernaemontana solanifolia A.DC. & $\mathrm{CD}^{*}$ & $\mathrm{BCF}$ \\
\hline Tassadia burchellii E.Fourn. & $\mathrm{CD}^{*}$ & \\
\hline Tassadia obovata Decne. & & BCF $^{*}$ \\
\hline Tassadia propinqua Decne. & & $\mathrm{BCF}^{*}$ \\
\hline Temnadenia odorifera (Vell.) J.F.Morales & & $\mathrm{BCF}^{*}$ \\
\hline Temnadenia violacea (Vell.) Miers & $\mathrm{CD}^{*}$ & $\mathrm{BCF}$ \\
\hline
\end{tabular}

material and analysed at relatively large scales. Ten thousand random (phylogenetically unstructured) communities were generated using the Apocynaceae pseudochronogram and a species pool from northeastern Brazil. This region is much larger than both $\mathrm{CD}$ and $\mathrm{BCF}$, but only approximately onequarter of the species in northeastern Brazil are not represented in either CD or BCF (Figure 1). To assess statistical differences in SR, PD, NRI, and NTI between CD and BCF, we used Kruskal-Wallis test and pairwise correlations between measures of diversity using Spearman's index, both in $\mathrm{R}$ statistical software [58]. To ensure that results of statistical tests are not overestimated due to spatial autocorrelation of analysis [59, 60], we also performed Kruskal-Wallis tests for SR, PD, and PS values without neighborhood cells at both spatial scales.

\section{Results}

Apocynaceae chronogram from matK sequences (Figure S1; see Supplementary Material available online at http://dx.doi .org/10.1155/2015/758019) mostly agree with the topology summarized in Rapini [47], based on several phylogenetic studies, and also with the most recent classification of the family [44]. The few hard conflicts (posterior probability $=100 \%$ ) were (1) Tylophora sister to AsclepiadinaeCynanchinae clade, rather than within Cynanchinae; (2) Macropharynx, Peltastes, and Temnadenia forming a clade without Prestonia, but agreeing with Peltastinae circumscription [44]; and (3) Ambelania sister to Tabernaemontana, making Ambelaniinae paraphyletic. The origin of Apocynaceae (stem group) was estimated at 68.56 million years 


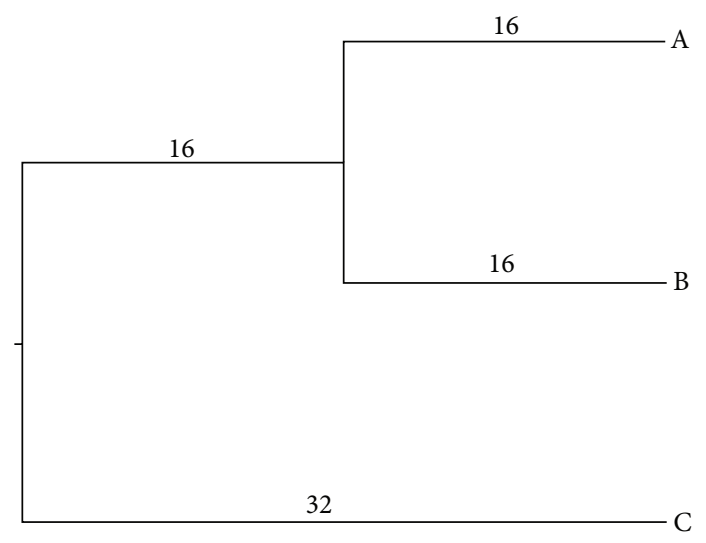

(a)

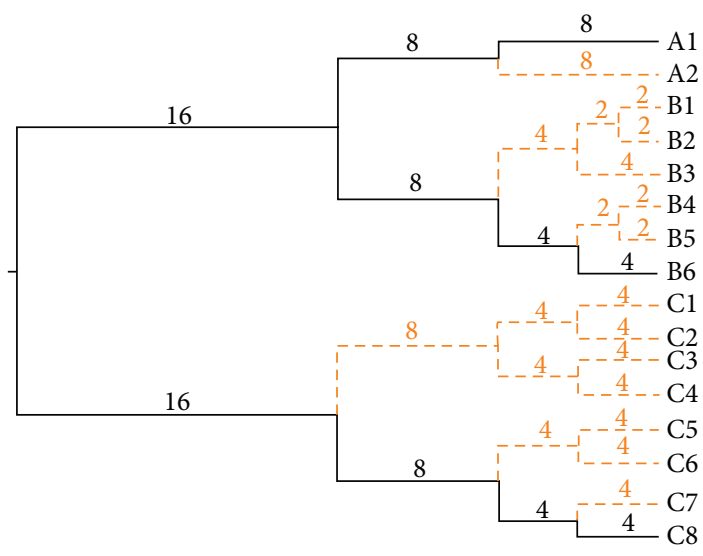

(b)

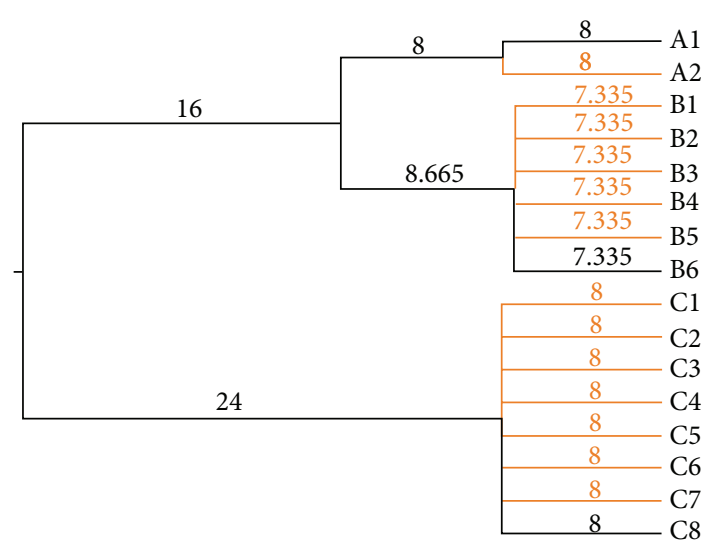

(c)

Figure 3: Procedure for calculating species' branch lengths. (a) Chronogram from Bayesian analysis. (b) Estimating lineage branch length: species lacking molecular data are inserted sequentially at the half length of the longest branches (dashed, orange branches). (c) Total branch length of the clade is standardized and species polytomized at the stem, which corresponds to the original age of lineage minus the species standardized branch length.

ago $(\mathrm{Ma}$; HPD $95 \%=55.92-84.4 \mathrm{Ma})$, of APSA clade at $50.52 \mathrm{Ma}$ (47.19-56.38), and of Asclepiadoideae at $27.35 \mathrm{Ma}$ (21.57-33.48). Genera estimates ranged from $36.83 \mathrm{Ma}(22.6-$ $52.9)$ for Rauvolfia to $4.8 \mathrm{Ma}(1.2-9.4)$ for the monotypic Hancornia (H. speciosa).

The Atlantic Forest domain shelters 130 species of Apocynaceae in northeastern Brazil and the Caatinga domain 154; 79 of these species occur in both domains. PD, estimated from the pseudochronogram (Figure S2), and SR were strongly correlated $\left(P \leq 2.2 e-16, r_{s}=0.945\right)$ and showed similar distributions in northeastern Brazil, with exceptionally high values concentrated in the CD and BCF (Figure 4). CD corresponds to only $4.5 \%$ of Caatinga in northeastern Brazil but shelters $77 \%$ of its SR (118 of 154 species) and $78 \%$ of its PD; 22\% (37 species) of SR from CD was not found elsewhere in Caatinga, representing $8 \%$ of a restricted PD. BCF corresponds to $41 \%$ of the northeastern Atlantic Forest and comprises $80 \%$ of its SR (103 of 130 species) and $88 \%$ of its PD; $44 \%$ (46 species) of SR from BCF is not found anywhere else in the northern Atlantic Forest, representing $21 \%$ of a restricted PD (Figure 1).
PDs in CD and BCF are not statistically different, but their SR and NRIs are (Table 2). Overall, the Apocynaceae are phylogenetically clustered (NRI $>0$ ) in CD and overdispersed $(\mathrm{NRI}<0)$ in BCF. Recent relationships are not evidently structured in either region and the difference between them is not significant at small spatial scale $\left(0.08^{\circ} \times 0.08^{\circ}\right)$ when neighbour cells are not considered; otherwise, the difference is significant (Table 2), with CD appearing to be clustered $(\mathrm{NTI}>0)$ only at a large spatial scale $\left(0.5^{\circ} \times 0.5^{\circ}\right)$ when neighbour cells are not considered and BCF tending toward overdispersion (NTI < 0) (Figure 5). SR correlates to PD but only correlates to NRI when $0.08^{\circ}$ cells is used in CD. SR is not statistically correlated to NTI and PD is not correlated to NRI or NTI in most cases (Table 3).

\section{Discussion}

The Apocynaceae have been phylogenetically investigated using several molecular regions (summarized in [47]) and, more recently, plastome analyses were also employed to resolve major relationships in the APSA clade [61]. Advances 


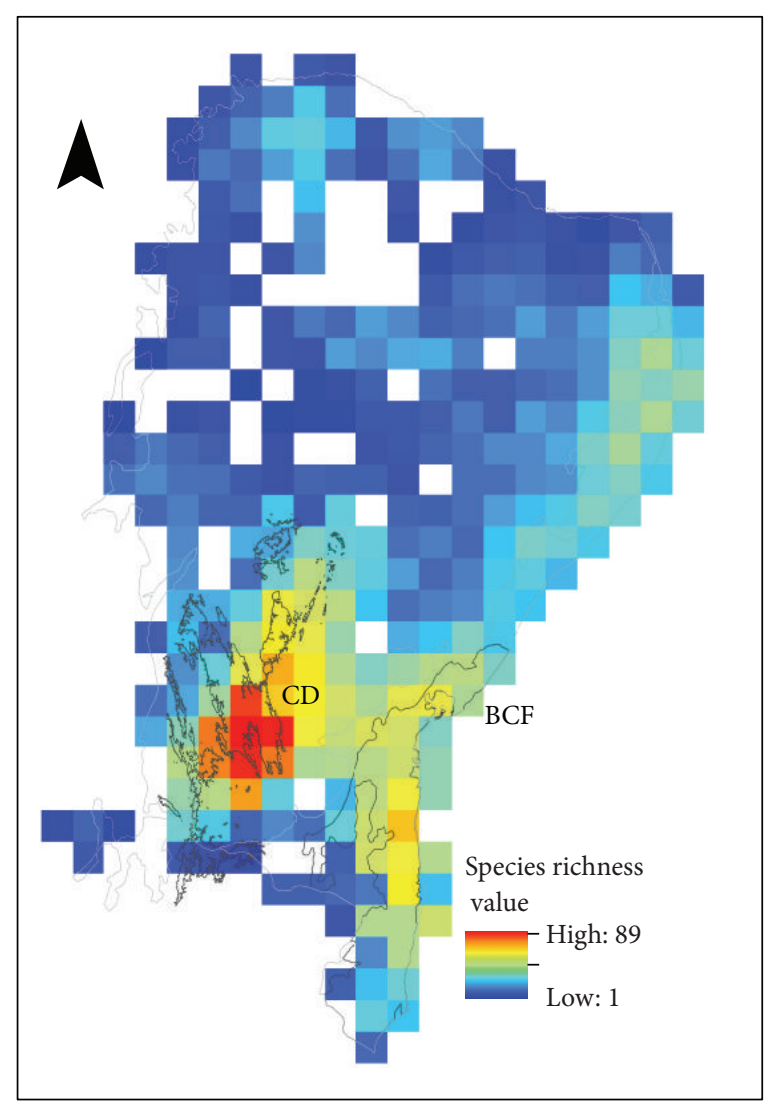

(a)

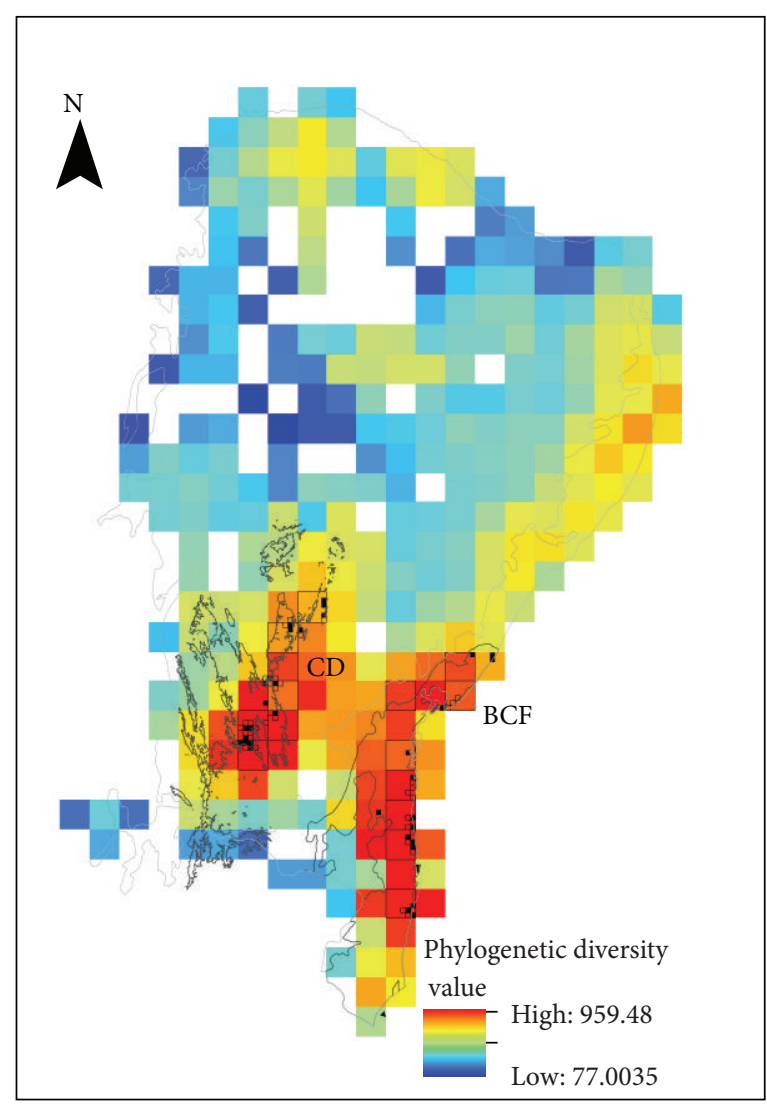

(b)

Figure 4: Distribution of species richness (a) and phylogenetic diversity (b) of Apocynaceae in northeastern Brazil, using $0.5^{\circ} \times 0.5^{\circ}$ cells with one neighborhood cell; $0.5^{\circ}$ cells with black margins present exceptionally high (5\% highest) phylogenetic diversity without neighborhood in Chapada Diamantina (CD) and Bahia Costal Forest (BCF); $0.08^{\circ}$ cells present exceptionally high (2.5\% highest) phylogenetic diversity with (black margins) and without neighborhood cells (black squares) in CD and BCF.

TABLE 2: Kruskal-Wallis test for species richness (SR), phylogenetic diversity (PD), net relatedness index (NRI), and nearest taxon index (NTI) in cells with exceptionally high PD in Chapada Diamantina and Bahia Costal Forest, using $0.5^{\circ} \times 0.5^{\circ}$ and $0.08^{\circ} \times 0.08^{\circ}$ cells, with $(\mathrm{Nc})$ and without $(\mathrm{wNc})$ one neighborhood cell.

\begin{tabular}{lcccccccc}
\hline & \multicolumn{2}{c}{$0.5^{\circ} \mathrm{Nc}$} & \multicolumn{2}{c}{$0.5^{\circ} \mathrm{wNc}$} & \multicolumn{2}{c}{$0.08^{\circ} \mathrm{Nc}$} & \multicolumn{2}{c}{$0.08^{\circ} \mathrm{wNc}$} \\
& $P$ & $x^{2}$ & $P$ & $x^{2}$ & $P$ & $x^{2}$ & 0.2744 \\
PD & 0.6256 & 0.2381 & 0.2976 & 1.0848 & 0.5311 & 0.3923 & 1.1946 \\
SR & 0.001 & 10.7407 & 0.03136 & 4.6333 & $3.49 E-05$ & 17.1326 & 0.0002 \\
NRI & 0.0006 & 11.6667 & 0.001194 & 10.5 & $7.43 E-07$ & 24.5 & $2.96 E-05$ & 13.3278 \\
NTI & 0.005 & 7.8107 & 0.007774 & 7.0848 & 0.005194 & 7.8107 & 0.08184 \\
\hline
\end{tabular}

in Apocynaceae systematics is incorporated into an updated classification at the tribal and subtribal levels [44], but many genera are not monophyletic (e.g., $[62,63]$ ) or still need a thorough phylogenetic investigation [47]. So far, dated phylogenies in Apocynaceae focused only on less inclusive groups, such as Asclepiadoideae [64], Tylophorinae [65], and Minaria [66], and used trnL intron and $\operatorname{trnL}-F$ intergenic space $(\operatorname{trn} L-F)$ for the Apocynaceae big picture. Therefore, this is the first Apocynaceae dated phylogeny using matK. Our results consistently recovered relationships obtained in previous studies and age estimates overlap those with $t r n L$ $F[66]$ when confidence intervals are taken into account.
However, the small sampling in major groups of Apocynaceae prevents a comprehensive biogeographic discussion.

Most of the Apocynaceae diversity in northeastern Brazil is concentrated in the Campos Rupestres of Chapada Diamantina (CD) and in the Bahia Costal Forest (BCF). CD and BCF are historical refuges for plants in two different floristic domains. CD is considered a refuge for grasslands during interglacial periods (e.g., $[34,67,68]$ ) or for firesensitive lineages after the expansion of the fire-prone Cerrado in Central Brazil $[36,66]$, whereas BCF is a refuge for forest associated lineages [40]. These plant refuges shelter an exceptionally high PD, which is phylogenetically clustered 

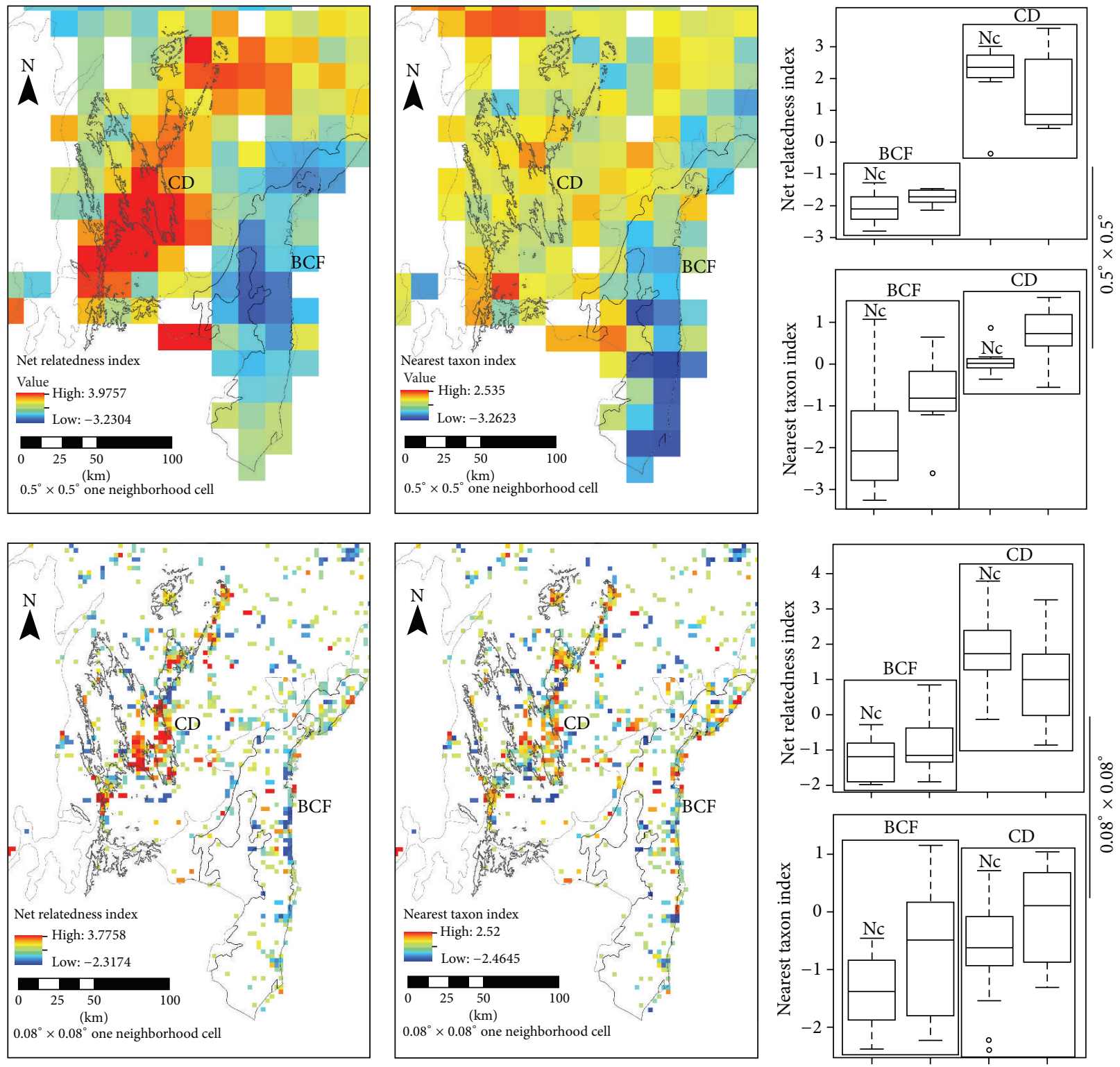

FIGURE 5: Distribution of net relatedness index (NRI) and nearest taxon index (NTI) in northeastern Brazil with bloxplots comparing 32 cells with exceptionally high phylogenetic diversity (Figure 4) in Chapada Diamantina (CD) and Bahia Costal Forest (BCF), using different scales $\left(0.5^{\circ} \times 0.5^{\circ}\right.$ and $0.08^{\circ} \times 0.08^{\circ}$ cells $)$, with $(\mathrm{Nc})$ and without one neighborhood cell.

TABLE 3: Spearman's correlation values for species richness (SR), phylogenetic diversity (PD), net relatedness index (NRI), and nearest taxon index (NTI) in cells with exceptionally high PD in the Bahia Costal Forest (BCF) and Chapada Diamantina $(\mathrm{CD}), \mathrm{using} 0.5^{\circ} \times 0.5^{\circ}$ and $0.08^{\circ} \times$ $0.08^{\circ}$ cells, with $(\mathrm{Nc})$ and without $(\mathrm{wNc})$ one neighborhood cell; asterisks indicate the significance values $(P<0.05)$.

\begin{tabular}{ccccccccccccc}
\hline & \multirow{2}{*}{ Scales } & \multicolumn{2}{c}{ SR-PD } & \multicolumn{4}{c}{ SR-NRI } & \multicolumn{2}{c}{ SR-NTI } & \multicolumn{2}{c}{ PD-NRI } & \multicolumn{2}{c}{ PD-NTI } \\
& & $P$ value & $r_{s}$ & $P$ value & $r_{s}$ & $P$ value & $r_{s}$ & $P$ value & $r_{s}$ & $P$ value & $r_{s}$ \\
\hline \multirow{4}{*}{$\mathrm{BCF} \quad$} & $0.5^{\circ} \mathrm{Nc}$ & $0.0003^{*}$ & 0.9 & 0.91 & 0.03 & 0.47 & 0.25 & 0.82 & -0.07 & 0.72 & 0.12 \\
& $0.5^{\circ} \mathrm{wNc}$ & $0.005^{*}$ & 0.86 & 0.79 & -0.1 & 0.12 & -0.59 & 0.38 & -0.35 & $0.028^{*}$ & -0.76 \\
& $0.08^{\circ} \mathrm{Nc}$ & $0.01^{*}$ & 0.62 & 0.15 & 0.4 & 0.7 & -0.11 & 0.28 & -0.3 & 0.74 & 0.09 \\
& $0.08^{\circ} \mathrm{wNc}$ & $0.019^{*}$ & 0.57 & 0.37 & 0.23 & 0.17 & 0.35 & 0.31 & -0.26 & 0.38 & -0.23 \\
\hline \multirow{4}{*}{$\mathrm{CD} \quad$} & $0.5^{\circ} \mathrm{Nc}$ & $0.002519^{*}$ & 0.92 & 0.7599 & 0.14 & 0.75 & -0.14 & 0.87 & -0.07 & 0.75 & -0.14 \\
& $0.5^{\circ} \mathrm{wNc}$ & $0.01^{*}$ & 0.85 & 0.21 & 0.53 & 0.75 & -0.14 & 0.38 & 0.39 & 0.39 & -0.39 \\
& $0.08^{\circ} \mathrm{Nc}$ & $3.50 E-09^{*}$ & 0.92 & 0.0007 & 0.67 & 0.64 & -0.1 & $5.89 E-05^{*}$ & 0.76 & 0.07 & -0.39 \\
& $0.08^{\circ} \mathrm{wNc}$ & $0.0002^{*}$ & 0.78 & 0.002 & 0.7 & 0.74 & 0.09 & 0.18 & 0.35 & 0.12 & -0.39 \\
\hline
\end{tabular}




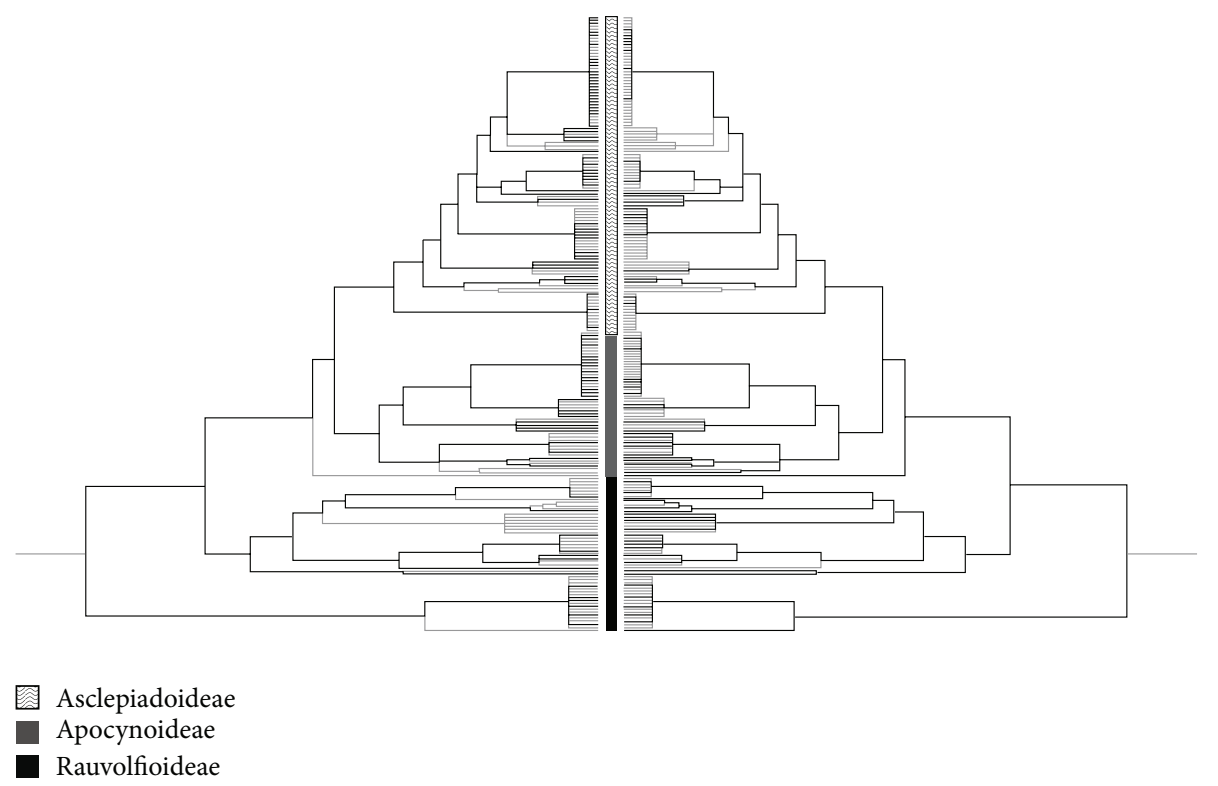

Figure 6: Backbone of Apocynaceae pseudochronogram (modified from Figure S2) showing the lineages represented in the exceptionally high PD cells of the Chapada Diamantina (left) and Bahia Costal Forest (right) in bold.

in CD but overdispersed in BCF. This difference is mirrored in SR, which is higher and spatially concentrated in CD. The Asclepiadoideae and the upper Apocynoideae grade are better represented in $\mathrm{CD}$, contributing to a high number of young lineages, while the Apocynaceae basal grade, including Rauvolfioideae and the early Apocynoideae, is better represented in $\mathrm{BCF}$, contributing to fewer but older lineages (Figure 6).

Plants in tropical forests have usually presented clustered phylogenies (e.g., [10, 20, 69-71]), contrasting with BCF. There are several potential explanations for this difference. First, most studies in tropical forests considered only tree communities, which tend to be phylogenetically clustered [30]. Second, their phylogenetic scale often comprises the whole angiosperms, and scales that are phylogenetically more inclusive are more likely to produce clustered PS [15-17] and also lose the power for predicting ecological processes as convergent traits increase to deeper relationships [3]. Third, such analyses are usually produced from poorly resolved phylogenies, in which species are unresolved within genera and genera within families. A lack of phylogenetic resolution also limits the analysis power and results can be incorrect, particularly near the tips, because many families are still awaiting phylogenetic analyses at the genus level.

In BCF, the overdispersed phylogeny at family scale is possibly produced by phenotypic repulsion caused by longterm competitive exclusion in a climatically stable region, rather than by phylogenetic attraction because of convergent traits, which is more likely at higher phylogenetic scales. When species are phylogenetically evenly distributed, as in BCF, niche overlap is expected to be reduced, and species probably have complementary fluctuations, responding differently to environmental changes and replacing one another in dominance, while maintaining ecosystem function. Because of that, diverse communities are more robust to species invasion, more productive and more resilient to environmental changes [12]. Under climatic changes, estimates are that in 50 years, neotropical ever-wet zones will be one-third smaller because of increasing seasonal variability in rainfall [72]. As such, refuges of biodiversity like $\mathrm{BCF}$-stable climatic region that is home of a high and phylogenetically evenly distributed evolutionary diversitydeserve high priority for in situ conservation.

The Apocynaceae are spatially and phylogenetically compact in CD, a pattern different from that in BFC. The overall clustering in $\mathrm{CD}$ reflects the heterogeneity and fragmented distribution of Campos Rupestres. At mountaintops, this biome consists of a mosaic of microhabitats at a small scale with an insular distribution at a large scale, resulting in high $\beta$ diversity [73]. Accordingly, the same spatial scales used in BCF tend to comprise more heterogeneous areas in $\mathrm{CD}$ and, therefore, are more likely to support species with different ecological traits in larger clades (e.g., Metastelmatinae) and also a higher number of allopatric, closelyrelated, microendemic species with similar ecological traits. This biogeographic pattern is reflected by the overall clustered PS in CD. For recent relationships, the phylogeny is not evidently structured, suggesting a stronger influence of neutral processes. Therefore, at a biogeographic context, deeper and narrower phylogenetic structures together suggest a stronger influence of niche conservatism, limited dispersal and in situ diversification in $\mathrm{CD}$, which can explain the high density of microendemic species in the Campos Rupestres as a result of nonadaptive, geographic radiations, as postulated by Ribeiro et al. ([36]; see also [66]).

According to the most popular hypothesis of diversification in the Espinhaço Range, the Campos Rupestres is cold associated, contracting to highlands during warmer periods 
and expanding to lowlands during cooler periods. Diversification resulted from successive contraction-expansion cycles caused by Pleistocene climatic fluctuations (e.g., [34, 67, 68]), as also suggested by refugial sites in different continents (e.g., [74, 75]). Alternatively, highlands represent refuges for fire-sensitive lineages and diversification was driven by the expansion of fire-prone Cerrado and fragmentation of Campos Rupestres since the late Miocene-Pliocene [36]. According to this hypothesis, milder weather and a high concentration of rocky outcrops in the highlands have helped to prevent frequent, intense fires, and diversification is result of a long-term contraction of the Campos Rupestres. Both scenarios consider mountaintops along the Espinhaço Range ecologically stable areas buffering biome conservative lineages during environmental changes and fit the PS recovered for Apocynaceae here.

Phylogenetic diversity and species relatedness reflect important properties for community function and stability $[12,76]$. The high PD in CD resulted from high SR of closely related species, in clustered structured communities; therefore, communities in $\mathrm{CD}$ are probably more vulnerable than in BCF. Past and future distribution models estimated a smaller distribution of Campos Rupestres today compared to in the Last Maximum Glacial and even smaller distributions in the future, almost disappearing in CD by the end of this century because of increasing seasonality [77]. Under this scenario, the reduction of the Campos Rupestres range is a natural process and loss of biodiversity in CD is probably inevitable. However, this process has been greatly accelerated by anthropogenic changes and CD may not represent a biodiversity refuge in the short future. Therefore, ex situ conservation is probably an important strategy to retain the biodiversity from the Campos Rupestres of the CD available in the future.

\section{Conclusion}

Ecology and phylogeny have been used independently from each other in biogeography [24]. Ecophylogenetics fills part of this gap and its use for investigating community structure has been increasing quickly [78]. However, ecophylogenetics and macroecology are still somewhat separate from each other, and few authors (e.g., [25, 79]) dared to analyse PS at large geographical scales. Although attention has been given more recently to species evolutionary distinctiveness, different components of a multifaceted biodiversity cannot be confidently used as surrogate of others (e.g., [80-82]).

The use of phylogenies through PD (sensu Faith [5]) in conservation goes beyond the traditional SR because it also includes evolutionary information. However, PD does not take into account species relationships and therefore misses an important aspect from which processes and factors that have driven community diversity can be inferred and functional diversity can be estimated [80]. PS emerges as a key concept in this context because it distributes PD across SR, shaping the evolutionary trace of a community, and, at the same time, provides indirect access for functional diversity, in particular when traits show a high phylogenetic signal. Thus, PS becomes an important measure to assess the vulnerability of communities against climatic changes or ecological and anthropogenic disturbances, providing information for conservation, and can be used for both understanding the past and anticipating the future.

SR, PD, and PS represent different aspects of biodiversity, and, together, they provide a more complete framework for conservation assessments. Our study shows that individual extinctions are probably more influent phylogenetically in $\mathrm{BCF}$ than in CD because $\mathrm{PD} / \mathrm{SR}$ ratio is higher in BCF. However, communities in BCF are usually overdispersed and, therefore, probably more resilient against invasive species, climatic changes, and anthropogenic disturbances than communities in $\mathrm{CD}$, which are phylogenetically unstructured or more often clustered. Based on PS pattern, different general conservation strategies can be designed; while in situ conservation may fit well for communities in BCF, an ex situ conservation is also recommended for protecting biodiversity in CD.

\section{Appendix}

\section{Taxa (Genbank Accession Number of matK Sequence)}

These include the following: Allamanda cathartica L. (Z70190); Acokanthera oblongifolia Benth. \& Hook.f. (Z70182); Allamanda schottii Pohl (DQ660495); Alstonia boonei De Wild. (KC627675); Alstonia scholaris (L.) R.Br. (AJ429321); Alyxia reinwardtii Blume (DQ660496); Alyxia oblongata Domin (EF456370); Ambelania acida Aubl. (DQ660497); Apocynum cannabinum L. (DQ660500); Apocynum androsaemifolium L. (Z70183); Araujia sericifera Brot. (Z98194); Asclepias syriaca L. (DQ660501); Asclepias curassavica L. (DQ026716); Aspidosperma cylindrocarpon Müll.Arg. (DQ660503); Aspidosperma australe Müll.Arg. (DQ660502); Baissea multiflora A.DC. (EF456319); Baissea zygodioides Stapf (DQ221120); Barjonia laxa Malme (JN805851); Barjonia cymosa E.Fourn. (JN805848); Barjonia erecta (Vell.) K.Schum. (JN805849); Blepharodon lineare (Decne.) Decne. (DQ026718); Blepharodon ampliflorum E.Fourn. (JN805852); Blepharodon nitidum (Vell.) J.F.Macbr. (DQ026720); Calotropis gigantea (L.) W.T.Aiton (JN228932); Carissa ovata R.Br. (DQ660506); Carissa macrocarpa A.DC. (DQ660505); Condylocarpon isthmicum (Vell.) A.DC. (DQ660511); Condylocarpon amazonicum (Markgr.) Ducke (DQ837537); Couma guianensis Aubl. (DQ660512); Cryptolepis sinensis Merr. (EF456374); Cryptostegia grandiflora R.Br. (Z98176); Cynanchum acutum L. (AY899939); Cynanchum auriculatum Buch.-Ham. ex Wight (GU373529); Ditassa banksii R.Br. ex Schult. (DQ026719); Ditassa auriflora Rapini (JN805856); Ditassa retusa Mart. (DQ026728); Dregea sinensis Hemsl. (Z98188); Dyera costulata Hook.f. (DQ660515); Farquharia elliptica Stapf (EF456357); Fockea capensis Endl. (Z98187); Fockea edulis K.Schum. (EF456383); Forsteronia velloziana (A.DC.) Woodson (DQ522596); Forsteronia acouci A.DC. (DQ522594); Funastrum clausum Schltr. (DQ026730); Galactophora schomburgkiana Woodson (EF456300); Geissospermum laeve Miers (DQ660517); 
Gomphocarpus fruticosus R.Br. (HM850833); Gonolobus xanthotrichus Brandegee (Z98195); Gymnanthera oblonga (Burm.f.) P.S.Green (EF456376); Hancornia speciosa Gomes (DQ660519); Hemipogon abietoides E.Fourn. (JN805868); Hemipogon luteus E.Fourn. (JN805874); Himatanthus bracteatus (A.DC.) Woodson (EF456366); Hunteria eburnea Pichon (DQ660521); Hunteria umbellata Hallier f. (KC627769); Hunteria zeylanica Gardner ex Thwaites (JX517717); Lacmellea aculeata (Ducke) Monach. (DQ660523); Lacmellea panamensis (Woodson) Markgr. (GQ982028); Logania vaginalis (Labill.) F.Muell. (AJ429324); Macoubea guianensis Aubl. (GU973901); Macroditassa grandiflora (E.Fourn.) Malme (JN805877); Macroditassa adnata Malme (JN805876); Macroditassa melantha (Silveira) Rapini (JN805879); Macropharynx renteriae A.H.Gentry (JQ586549); Macroscepis hirsuta (Vahl) Schltr. (JQ586771); Malouetia tamaquarina A.DC. (EF456346); Malouetia bequaertiana Woodson (EF456358); Mandevilla hirsuta (Rich.) K.Schum. (DQ522614); Mandevilla syrinx Woodson (DQ522637); Marsdenia engleriana W.Rothe (JQ586772); Melodinus australis (F.Muell.) Pierre (DQ660524); Melodinus cochinchinensis (Lour.) Merr. (DQ660525); Mesechites mansoanus (A.DC.) Woodson (DQ522644); Mesechites roseus (A.DC.) Miers (DQ522646); Metastelma schaffneri A.Gray (JN805884); Metastelma parviflorum (Sw.) Schult. (JN805883); Minaria harleyi (Fontella \& Marquete) Rapini \& U.C.S.Silva (JN805850); Minaria volubilis Rapini \& U.C.S.Silva (JN805871); Minaria grazielae (Fontella \& Marquete) T.U.P.Konno \& Rapini (DQ026724); Mitreola petiolata (J.F.Gmel.) Torr. \& A.Gray (JQ588153); Molongum laxum (Benth.) Pichon (Z70185); Mondia ecornuta (N.E.Br.) Bullock (AY899941); Motandra guineensis A.DC. (DQ221121); Mucoa duckei (Markgr.) Zarucchi (GU973902); Nautonia nummularia Decne. (JN805886); Neocouma ternstroemiacea (Müll.Arg.) Pierre (GU973903); Nephradenia filipes Malme (JN805889); Nephradenia asparagoides E.Fourn (JN805888); Nephradenia acerosa Decne. (JN805887); Nerium oleander L. (Z98173); Odontadenia lutea (Vell.) Markgr. (DQ522648); Odontadenia perrottetii (A.DC.) Woodson (EF456272); Oncinotis tenuiloba Stapf (DQ660529); Orthosia scoparia (Nutt.) Liede \& Meve (KF539851); Oxypetalum sublanatum Malme (DQ026738); Oxypetalum banksii Schult. (DQ026735); Parahancornia fasciculata (Poir.) Benoist (DQ660530); Peltastes isthmicus Woodson (EF456301); Peltastes peltatus (Vell.) Woodson (DQ660532); Pentopetia grevei (Baill.) Venter (AY899943); Peplonia macrophylla (Malme) U.C.S.Silva \& Rapini (JN805878); Peplonia organensis (E.Fourn.) Fontella \& Rapini (JN805891); Peplonia asteria (Vell.) Fontella \& E.A.Schwarz (JN805890); Periploca graeca L. (Z98178); Pervillaea phillipsonii Klack. (AJ312408); Pervillaea venenata (Baill.) Klack. (Z98181); Petalostelma martianum E.Fourn. (JN805892); Petalostelma sarcostemma (Lillo) Liede \& Meve (JN805893); Petopentia natalensis (Schltr.) Bullock (DQ660533); Philibertia discolor (Schltr.) Goyder (DQ026732); Philibertia lysimachioides (Wedd.) T.Mey. (DQ026741); Phyllanthera grayi (P.I.Forst.) Venter (DQ660534); Pleiocarpa pycnantha Stapf (JX517964); Pleiocarpa mutica Benth. (DQ660535); Pleiocarpa rostrata Benth. (KC627551); Prestonia lagoensis Woodson (EF456329);
Prestonia mexicana A.DC. (EF456345); Raphionacme flanaganii Schltr. (EF456377); Raphionacme welwitschii Schltr. \& Rendle (Z98179); Rauvolfia vomitoria Wennberg (DQ660538); Rauvolfia mannii Stapf (Z70181); Rhabdadenia biflora Müll.Arg. (EF456277); Rhabdadenia macrostoma Müll.Arg. (EF456349); Rhigospira quadrangularis (Müll.Arg.) Miers (GU973904); Secamone elliptica R.Br. (DQ660541); Secamone bosseri Klack. (Z98182); Secamone geayi Costantin \& Gallaud (Z98184); Secamonopsis madagascariensis Jum. (Z98185); Secondatia densiflora A.DC. (DQ522653); Spigelia anthelmia L. (JQ588156); Spongiosperma macrophyllum (Müll.Arg.) Zarucchi (GU973905); Stapelia gigantea N.E.Br. (JQ025000); Stephanostema stenocarpum K.Schum. (DQ660543); Stipecoma peltigera Müll.Arg. (EF456314); Strychnos axillaris Colebr. (AB636276); Tabernaemontana cuspidata Rusby (GU973931); Tabernaemontana hystrix Steud. (GU973942); Tacazzea apiculata Oliv. (AY899945); Temnadenia violacea (Vell.) Miers (EF456341); Temnadenia odorifera (Vell.) J.F.Morales (EF456353); Thevetia peruviana K.Schum. (Z70188); Thevetia ahouai A.DC. (GQ982112); Toxocarpus villosus Decne. (EF456379); Tylophora indica Merr. (Z98193); Vallesia antillana Woodson (AM295075); Wrightia dubia Spreng. (EF456257); Wrightia lanceolata Kerr (EF456291).

\section{Conflict of Interests}

The authors declare that there is no conflict of interests regarding the publication of this paper.

\section{Acknowledgments}

This study is part of the M.Sc. thesis of Lara Pugliesi, which was developed at PPGBot-UEFS with a fellowship from CNPq. Alessandro Rapini is supported by Pq-1D CNPq grant. The authors also thank $\mathrm{CNPq}$ for supporting the project on "Phyloconservation" (no. 485468/2013-1).

\section{References}

[1] B. J. Cardinale, J. E. Duffy, A. Gonzalez et al., "Biodiversity loss and its impact on humanity," Nature, vol. 486, no. 7401, pp. 5967, 2012.

[2] F. Forest, R. Grenyer, M. Rouget et al., "Preserving the evolutionary potential of floras in biodiversity hotspots," Nature, vol. 445, no. 7129, pp. 757-760, 2007.

[3] J. Cavender-Bares, K. H. Kozak, P. V. A. Fine, and S. W. Kembel, "The merging of community ecology and phylogenetic biology," Ecology Letters, vol. 12, no. 7, pp. 693-715, 2009.

[4] R. I. Vane-Wright, C. J. Humphries, and P. H. Williams, "What to protect?-Systematics and the agony of choice," Biological Conservation, vol. 55, no. 3, pp. 235-254, 1991.

[5] D. P. Faith, "Conservation evaluation and phylogenetic diversity," Biological Conservation, vol. 61, no. 1, pp. 1-10, 1992.

[6] D. P. Faith, "Phylogenetic diversity and conservation," in Conservation Biology, S. P. Carrol and C. W. Fox, Eds., pp. 99-115, Oxford University Press, New York, NY, USA, 2008. 
[7] D. P. Faith and A. M. Baker, "Phylogenetic diversity (PD) and biodiversity conservation: some bioinformatics challenges," Evolutionary Bioinformatics Online, vol. 2, pp. 121-128, 2006.

[8] S. B. Heard and A. Ò. Mooers, "Phylogenetically patterned speciation rates and extinction risks change the loss of evolutionary history during extinctions," Proceedings of the Royal Society B: Biological Sciences, vol. 267, no. 1443, pp. 613-620, 2000.

[9] A. Ø. Mooers and S. B. Heard, "Evolutionary heritage as a metric for conservation," in Phylogeny and Conservation, A. Purvis, J. L. Gittleman, and T. Brooks, Eds., pp. 120-138, Cambridge University Press, Cambridge, UK, 2005.

[10] C. O. Webb, "Exploring the phylogenetic structure of ecological communities: an example for rain forest trees," The American Naturalist, vol. 156, no. 2, pp. 145-155, 2000.

[11] C. O. Webb, D. D. Ackerly, M. A. McPeek, and M. J. Donoghue, "Phylogenies and community ecology," Annual Review of Ecology and Systematics, vol. 33, pp. 475-505, 2002.

[12] N. Mouquet, V. Devictor, C. N. Meynard et al., "Ecophylogenetics: advances and perspectives," Biological Reviews of the Cambridge Philosophical Society, vol. 87, no. 4, pp. 769-785, 2012.

[13] M. D. Crisp, M. T. K. Arroyo, L. G. Cook et al., "Phylogenetic biome conservatism on a global scale," Nature, vol. 458, no. 7239, pp. 754-756, 2009.

[14] M. D. Crisp and L. G. Cook, "Phylogenetic niche conservatism: what are the underlying evolutionary and ecological causes?" New Phytologist, vol. 196, no. 3, pp. 681-694, 2012.

[15] J. Cavender-Bares, A. Keen, and B. Miles, "Phylogenetic structure of Floridian plant communities depends on taxonomic and spatial scale," Ecology, vol. 87, no. 7, pp. S109-S122, 2006.

[16] N. G. Swenson, B. J. Enquist, J. Pither, J. Thompson, and J. K. Zimmerman, "The problem and promise of scale dependency in community phylogenetics," Ecology, vol. 87, no. 10, pp. 24182424, 2006.

[17] N. G. Swenson, B. J. Enquist, J. Thompson, and J. K. Zimmerman, "The influence of spatial and size scale on phylogenetic relatedness in tropical forest communities," Ecology, vol. 88, no. 7, pp. 1770-1780, 2007.

[18] B. C. Emerson and R. G. Gillespie, "Phylogenetic analysis of community assembly and structure over space and time," Trends in Ecology and Evolution, vol. 23, no. 11, pp. 619-630, 2008.

[19] S. M. Vamosi, S. B. Heard, J. C. Vamosi, and C. O. Webb, "Emerging patterns in the comparative analysis of phylogenetic community structure," Molecular Ecology, vol. 18, no. 4, pp. 572592, 2009.

[20] S. W. Kembel and S. P. Hubbell, "The phylogenetic structure of a neotropical forest tree community," Ecology, vol. 87, no. 7, pp. S86-S99, 2006.

[21] M. R. Helmus, T. J. Bland, C. K. Williams, and A. R. Ives, "Phylogenetic measures of biodiversity," The American Naturalist, vol. 169, no. 3, pp. E68-E83, 2007.

[22] O. J. Hardy, “Testing the spatial phylogenetic structure of local communities: statistical performances of different null models and test statistics on a locally neutral community," Journal of Ecology, vol. 96, no. 5, pp. 914-926, 2008.

[23] M. Winter, V. Devictor, and O. Schweiger, "Phylogenetic diversity and nature conservation: where are we?" Trends in Ecology \& Evolution, vol. 28, no. 4, pp. 199-204, 2013.

[24] J. J. Wiens and M. J. Donoghue, "Historical biogeography, ecology and species richness," Trends in Ecology \& Evolution, vol. 19, no. 12, pp. 639-644, 2004.
[25] W. D. Kissling, W. L. Eiserhardt, W. J. Baker et al., "Cenozoic imprints on the phylogenetic structure of palm species assemblages worldwide," Proceedings of the National Academy of Sciences of the United States of America, vol. 109, no. 19, pp. 7379-7384, 2012.

[26] A. K. Brunbjerg, J. Cavender-Bares, W. L. Eiserhardt et al., "Multi-scale phylogenetic structure in coastal dune plant communities across the globe," Journal of Plant Ecology, vol. 7, no. 2, pp. 101-114, 2014.

[27] M. N. Umaña, N. Norden, Á. Cano, and P. R. Stevenson, "Determinants of plant community assembly in a mosaic of landscape units in Central Amazonia: ecological and phylogenetic perspectives," PLoS ONE, vol. 7, no. 9, Article ID e45199, 2012.

[28] Y. Yan, X. Yang, and Z. Tang, "Patterns of species diversity and phylogenetic structure of vascular plants on the QinghaiTibetan Plateau," Ecology and Evolution, vol. 3, no. 13, pp. 45844595, 2013.

[29] X.-X. Mo, L.-L. Shi, Y.-J. Zhang, H. Zhu, and J. W. F. Slik, "Change in phylogenetic community structure during succession of traditionally managed tropical rainforest in Southwest China," PLoS ONE, vol. 8, no. 7, Article ID e71464, 2013.

[30] H. Qian, Z. Hao, and J. Zhang, "Phylogenetic structure and phylogenetic diversity of angiosperm assemblages in forests along an elevational gradient in Changbaishan, China," Journal of Plant Ecology, vol. 7, no. 2, pp. 154-165, 2014.

[31] R. C. Forzza, A. Costa, B. M. T. Walter et al., "Angiospermas," Lista de Espécies da Flora do Brasil, 2014, http://floradobrasil .jbrj.gov.br/jabot/floradobrasil/FB128482.

[32] L. S. Duarte, R. S. Bergamin, V. Marcilio-Silva, G. D. S. Seger, and M. C. M. Marques, "Phylobetadiversity among forest types in the Brazilian Atlantic Forest complex," PLoS ONE, vol. 9, no. 8, Article ID e105043, 2014.

[33] A. A. de Oliveira, A. Vicentini, J. Chave et al., "Habitat specialization and phylogenetic structure of tree species in a coastal Brazilian white-sand forest," Journal of Plant Ecology, vol. 7, no. 2, pp. 134-144, 2014.

[34] A. Rapini, P. L. Ribeiro, S. Lambert, and J. R. Pirani, "A flora dos campos rupestres da Cadeia do Espinhaço," Megadiversidade, vol. 4, pp. 16-24, 2008.

[35] C. Bitencourt and A. Rapini, "Centres of endemism in the espinhaço range: identifying cradles and museums of Asclepiadoideae (Apocynaceae)," Systematics and Biodiversity, vol. 11, no. 4, pp. 525-536, 2013.

[36] P. L. Ribeiro, A. Rapini, U. C. S. E. Silva, T. U. P. Konno, L. S. Damascena, and C. V. D. Berg, "Spatial analyses of the phylogenetic diversity of Minaria (Apocynaceae): assessing priority areas for conservation in the Espinhaco Range, Brazil," Systematics and Biodiversity, vol. 10, no. 3, pp. 317-331, 2012.

[37] D. M. Olson, E. Dinerstein, E. D. Wikramanayake et al., "Terrestrial ecoregions of the world: a new map of life on earth," BioScience, vol. 51, no. 11, pp. 933-938, 2001.

[38] A. C. Carnaval and C. Moritz, "Historical climate modelling predicts patterns of current biodiversity in the Brazilian Atlantic forest," Journal of Biogeography, vol. 35, no. 7, pp. 1187-1201, 2008.

[39] T. J. Porto, A. C. Carnaval, and P. L. B. da Rocha, "Evaluating forest refugial models using species distribution models, model filling and inclusion: a case study with 14 Brazilian species," Diversity and Distributions, vol. 19, no. 3, pp. 330-340, 2013.

[40] A. C. Carnaval, E. Waltari, M. T. Rodrigues et al., "Prediction of phylogeographic endemism in an environmentally complex 
biome," Proceedings of the Royal Society B: Biological Sciences, vol. 281, no. 1792, 2014.

[41] W. W. Thomas, A. M. V. de Carvalho, A. M. A. Amorim, J. Garrison, and A. L. Arbeláez, "Plant endemism in two forests in southern Bahia, Brazil," Biodiversity and Conservation, vol. 7, no. 3, pp. 311-322, 1998.

[42] A. M. Z. Martini, P. Fiaschi, A. M. Amorim, and J. L. da Paixão, "A hot-point within a hot-spot: a high diversity site in Brazil's Atlantic Forest," Biodiversity and Conservation, vol. 16, no. 11, pp. 3111-3128, 2007.

[43] M. E. Endress, “Apocynaceae: brown and now," Telopea, vol. 10, pp. 525-541, 2004.

[44] M. E. Endress, S. Liede-Schumann, and U. Meve, "An updated classification for Apocynaceae," Phytotaxa, vol. 159, no. 3, pp. 175-194, 2014.

[45] I. Koch, A. Rapini, L. S. Kinoshita, A. O. Simões, and A. P. Spina, "Apocynaceae," in Lista de Espécies da Flora do Brasil, 2014, http://floradobrasil.jbrj.gov.br/jabot/floradobrasil/FB48.

[46] A. Rapini, "Apocynaceae," in Flowering Plants of the Neotropics, N. Smith, S. A. Mori, A. Henderson, D. W. Stevenson, and S. V. Head, Eds., pp. 23-26, Princeton University Press, Princeton, NJ, USA, 2004.

[47] A. Rapini, “Taxonomy 'under construction': advances in the systematics of Apocynaceae, with emphasis on the Brazilian Asclepiadoideae," Rodriguésia, vol. 63, no. 1, pp. 75-88, 2012.

[48] R. C. Edgar, "MUSCLE: multiple sequence alignment with high accuracy and high throughput," Nucleic Acids Research, vol. 32, no. 5, pp. 1792-1797, 2004.

[49] W. P. Maddison and D. R. Maddison, Mesquite: A Modular System for Evolutionary Analysis. Version 2.7, 2010, http://mesquiteproject.org/.

[50] A. J. Drummond, M. A. Suchard, D. Xie, and A. Rambaut, "Bayesian phylogenetics with BEAUti and the BEAST 1.7," Molecular Biology and Evolution, vol. 29, no. 8, pp. 1969-1973, 2012.

[51] M. A. Miller, W. Pfeiffer, and T. Schwartz, "Creating the CIPRES science gateway for inference of large phylogenetic trees," in Proceedings of the Gateway Computing Environments Workshop (GCE '10), pp. 1-8, New Orleans, La, USA, November 2010.

[52] M. E. Collinson, S. R. Manchester, V. Wilde, and P. Hayes, "Fruit and seed floras from exceptionally preserved biotas in the European Paleogene," Bulletin of Geosciences, vol. 85, no. 1, pp. 155-162, 2010.

[53] J. Muller, "Fossil pollen records of extant angiosperms," The Botanical Review, vol. 47, no. 1, pp. 1-142, 1981.

[54] A. J. Drummond and A. Rambaut, "BEAST: bayesian evolutionary analysis by sampling trees," BMC Evolutionary Biology, vol. 7, no. 1, article 214, 2007.

[55] S. W. Laffan, E. Lubarsky, and D. F. Rosauer, "Biodiverse, a tool for the spatial analysis of biological and related diversity," Ecography, vol. 33, no. 4, pp. 643-647, 2010.

[56] C. O. Webb, D. D. Ackerly, and S. W. Kembel, "Phylocom: software for the analysis of phylogenetic community structure and trait evolution," Bioinformatics, vol. 24, no. 18, pp. 20982100, 2008.

[57] C. O. Webb, D. D. Ackerly, and S. W. Kembel, "Phylocom: software for the analysis of phylogenetic community structure and trait evolution," Bioinformatics, vol. 24, no. 18, pp. 20982100, 2008.

[58] R Development Core Team, "R: a language and environmental for statistical computing," 2014, http://www.R-project.org.
[59] P. Legendre, "Spatial autocorrelation: trouble or new paradigm?” Ecology, vol. 74, no. 6, pp. 1659-1673, 1993.

[60] J. W. H. Ferguson and M. N. Bester, "The treatment of spatial autocorrelation in biological surveys: the case of line transect surveys," Antarctic Science, vol. 14, no. 2, pp. 115-122, 2002.

[61] S. C. Straub, M. J. Moore, P. S. Soltis, D. E. Soltis, A. Liston, and T. Livshultz, "Phylogenetic signal detection from an ancient rapid radiation: effects of noise reduction, long-branch attraction, and model selection in crown clade Apocynaceae," Molecular Phylogenetics and Evolution, vol. 80, pp. 169-185, 2014.

[62] U. C. Soares e Silva, A. Rapini, S. Liede-Schumann, P. L. Ribeiro, and C. van den Berg, "Taxonomic considerations on Metastelmatinae (Apocynaceae) based on plastid and nuclear DNA," Systematic Botany, vol. 37, no. 3, pp. 795-806, 2012.

[63] S. Liede-Schumann, M. Nikolaus, U. C. S. Soares e Silva, A. Rapini, R. D. Mangelsdorff, and U. Meve, "Phylogenetics and biogeography of the genus Metastelma (ApocynaceaeAsclepiadoideae-Asclepiadeae: Metastelmatinae)," Systematic Botany, vol. 39, no. 2, pp. 594-612, 2014.

[64] A. Rapini, C. Van Den Berg, and S. Liede-Schumann, "Diversification of Asclepiadoideae (Apocynaceae) in the New World," Annals of the Missouri Botanical Garden, vol. 94, no. 2, pp. 407422, 2007.

[65] S. Liede-Schumann, H. Kong, U. Meve, and M. Thiv, "Vincetoxicum and Tylophora (Apocynaceae: Asclepiadoideae: Asclepiadeae)-two sides of the same medal: independent shifts from tropical to temperate habitats," Taxon, vol. 61, no. 4, pp. 803-825, 2012.

[66] P. L. Ribeiro, A. Rapini, L. S. Damascena, and C. van den Berg, "Plant diversification in the Espinhaço Range: insights from the biogeography of Minaria (Apocynaceae)," Taxon, vol. 63, no. 6, pp. 1253-1264, 2014.

[67] R. M. Harley, "Evolution and distribution of Eriope (Labiatae), and its relatives, in Brazil," in Proceedings of a Workshop on Neotropical Distribution Patterns, P. E. Vanzolini and E. R. Heyer, Eds., pp. 71-120, Academia Brasileira de Ciências, Rio de Janeiro, Brazil, 1988.

[68] R. M. Harley, "Introdução," in Flora of Pico das AlmasChapada Diamantina, Bahia, Brasil, B. L. Stannard, Ed., pp. 4348, Royal Botanic Gardens, Kew, UK, 1995.

[69] J. Chave, G. Chust, and C. Thébaud, "The importance of phylogenetic structure in biodiversity studies," in Scaling Biodiversity, D. Storch, P. Marquet, and J. Brown, Eds., pp. 150-167, Cambridge University Press, Cambridge, UK, 2007.

[70] O. J. Hardy and B. Senterre, "Characterizing the phylogenetic structure of communities by an additive partitioning of phylogenetic diversity," Journal of Ecology, vol. 95, no. 3, pp. 493-506, 2007.

[71] C. Baraloto, O. J. Hardy, C. E. T. Paine et al., "Using functional traits and phylogenetic trees to examine the assembly of tropical tree communities," Journal of Ecology, vol. 100, no. 3, pp. 690701, 2012.

[72] E. C. Underwood, D. Olson, A. D. Hollander, and J. F. Quinn, "Ever-wet tropical forests as biodiversity refuges questions of bias in climate models," Nature Climate Change, vol. 4, no. 9, pp. 740-741, 2014.

[73] A. A. Conceição, A. Rapini, J. R. Pirani et al., "Campos rupestres," in Biodiversidade e Conservação da Chapada Diamantina, F. A. Juncá, L. Funch, and W. Rocha, Eds., pp. 153-180, Ministério do Meio Ambiente, Brasília, Brazil, 2005. 
[74] J. K. Morton, "Phytogeography of the West African Mountains," in Taxonomy, Phytogeography and Evolution, D. H. Valentine, Ed., pp. 221-236, Academic Press, London, UK, 1972.

[75] P. C. Tzedakis, I. T. Lawson, M. R. Frogley, G. M. Hewitt, and R. C. Preece, "Buffered tree population changes in a Quaternary refugium: evolutionary implications," Science, vol. 297, no. 5589, pp. 2044-2047, 2002.

[76] M. W. Cadotte, R. Dinnage, and D. Tilman, "Phylogenetic diversity promotes ecosystem stability," Ecology, vol. 93, no. 8, pp. S223-S233, 2012.

[77] C. Bitencourt, Biogeografia das Asclepiadoideae (Apocynaceae) na Cadeia do Espinhaço: o futuro incerto dos refúgios glaciais de Campos Rupestres [M.S. thesis], Universidade Estadual de Feira de Santana, 2013.

[78] H. Qian and L. Jiang, "Phylogenetic community ecology: integrating community ecology and evolutionary biology," Journal of Plant Ecology, vol. 7, no. 2, pp. 97-100, 2014.

[79] M. Cardillo, "Phylogenetic structure of mammal assemblages at large geographical scales: linking phylogenetic community ecology with macroecology," Philosophical Transactions of the Royal Society B: Biological Sciences, vol. 366, no. 1577, pp. 25452553, 2011.

[80] V. Devictor, D. Mouillot, C. Meynard, F. Jiguet, W. Thuiller, and N. Mouquet, "Spatial mismatch and congruence between taxonomic, phylogenetic and functional diversity: the need for integrative conservation strategies in a changing world," Ecology Letters, vol. 13, no. 8, pp. 1030-1040, 2010.

[81] K. Safi, M. V. Cianciaruso, R. D. Loyola, D. Brito, K. ArmourMarshall, and J. A. F. Diniz-Filho, "Understanding global patterns of mammalian functional and phylogenetic diversity," Philosophical Transactions of the Royal Society B: Biological Sciences, vol. 366, no. 1577, pp. 2536-2544, 2011.

[82] F. Mazel, F. Guilhaumon, N. Mouquet et al., "Multifaceted diversity-area relationships reveal global hotspots of mammalian species, trait and lineage diversity," Global Ecology and Biogeography, vol. 23, no. 8, pp. 836-847, 2014. 

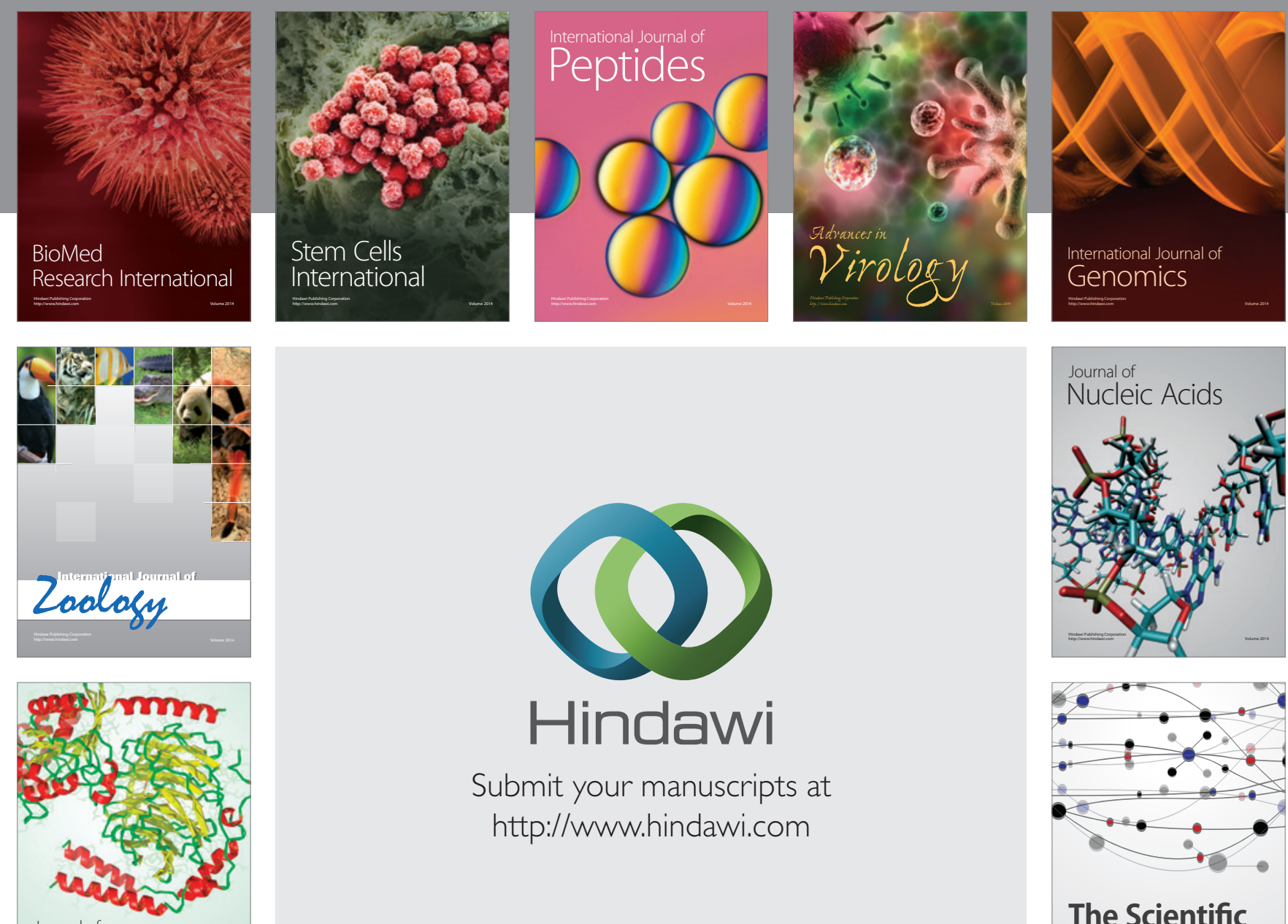

Submit your manuscripts at

http://www.hindawi.com

Journal of
Signal Transduction
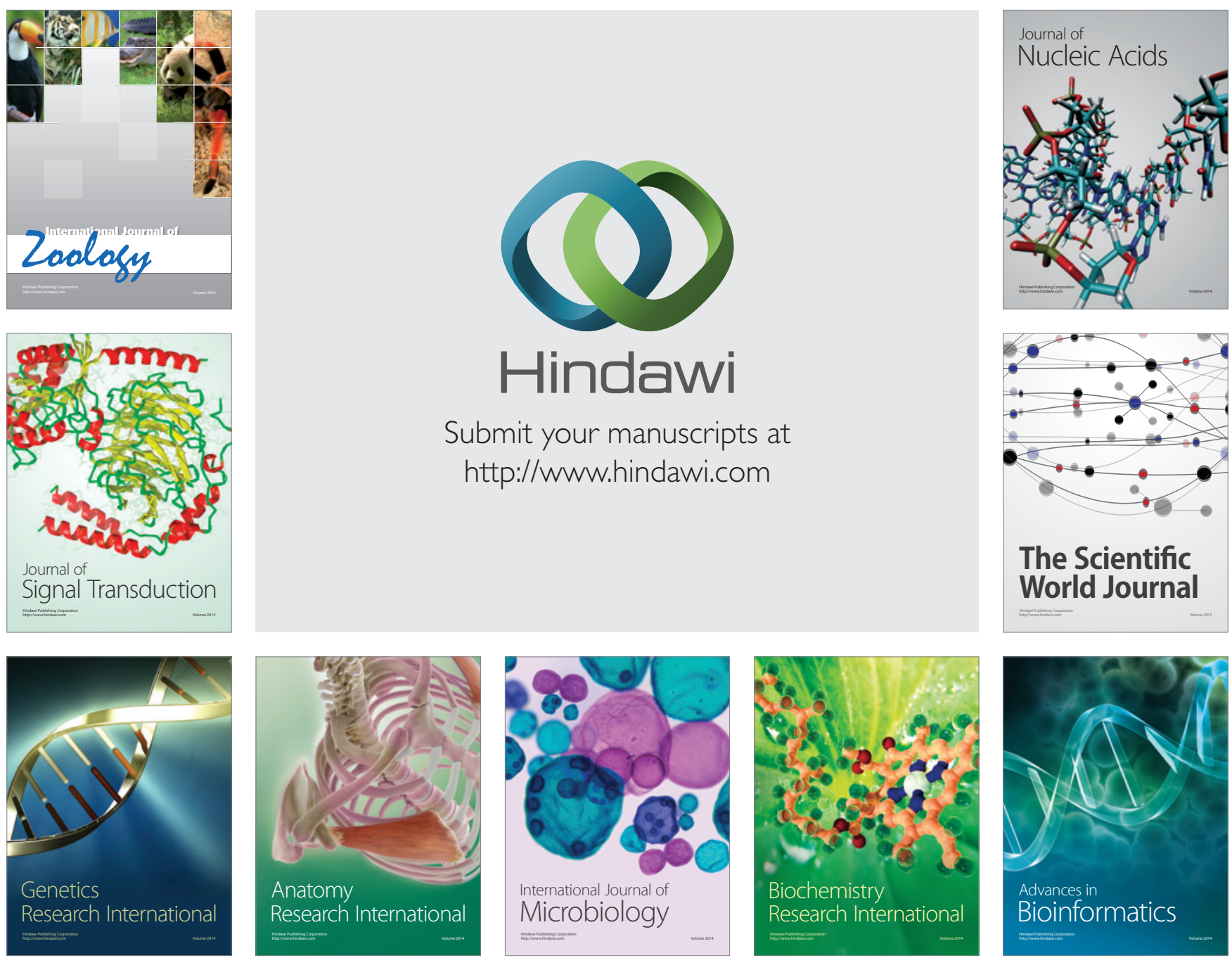

The Scientific World Journal
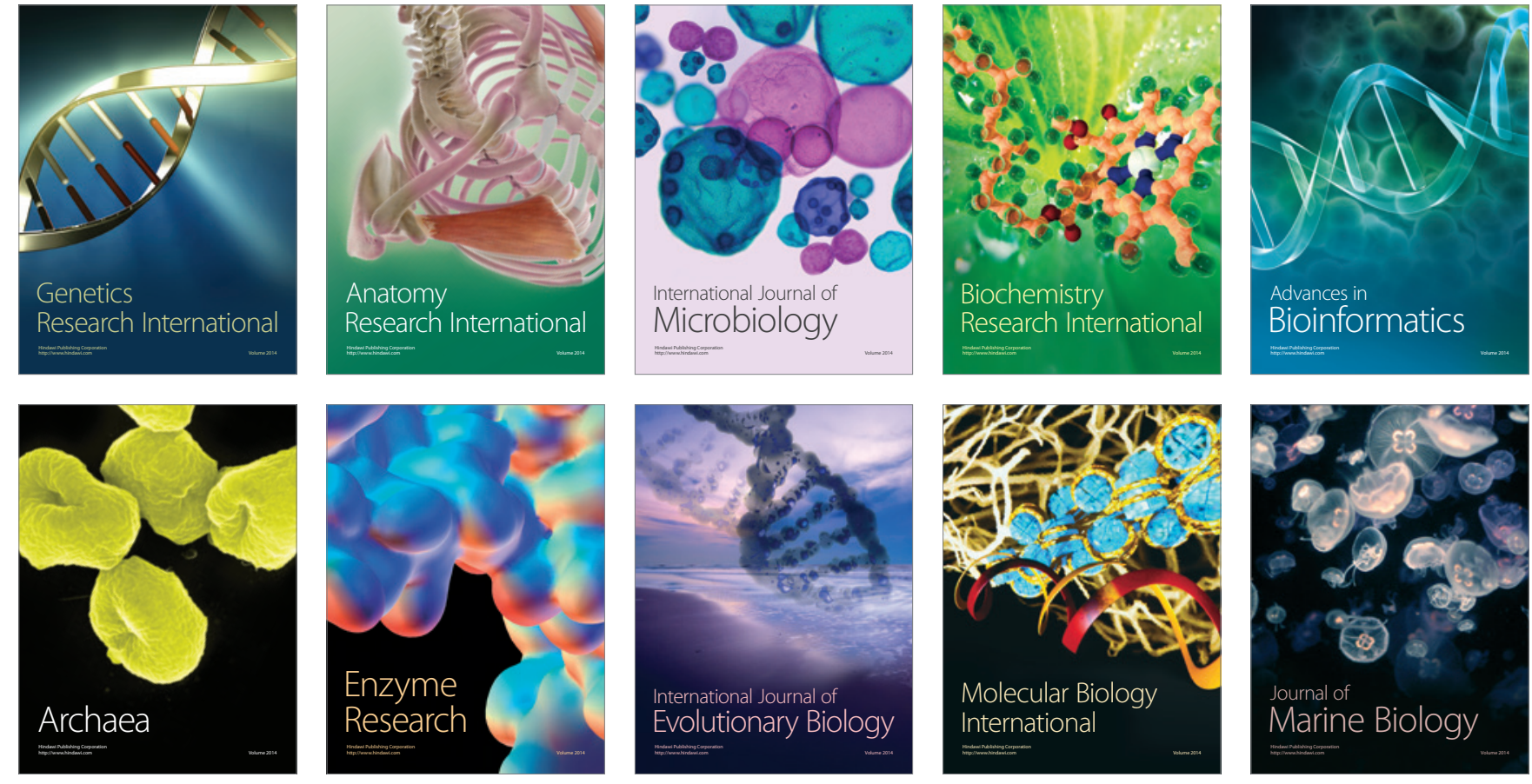